\title{
Art mobilier inédit du gisement de Bourrouilla à Arancou (Pyrénées-Atlantiques, France) : données techno-stylistiques et chrono- culturelles
}

Unpublished mobile art from Bourrouilla cave in Arancou (Pyrénées-

Atlantiques, France) : techno-stylistic and chronocultural data

Lise Aurière, François-Xavier Chauvière, Frédéric Plassard, Carole Fritz et Morgane Dachary

\section{OpenEdition \\ Journals}

Édition électronique

URL : http://journals.openedition.org/paleo/2624

DOI : $10.4000 /$ paleo.2624

ISSN : 2101-0420

Éditeur

SAMRA

Édition imprimée

Date de publication : 15 décembre 2013

Pagination : 192-217

ISSN : $1145-3370$

Référence électronique

Lise Aurière, François-Xavier Chauvière, Frédéric Plassard, Carole Fritz et Morgane Dachary, «Art mobilier inédit du gisement de Bourrouilla à Arancou (Pyrénées-Atlantiques, France) : données technostylistiques et chrono-culturelles », PALEO [En ligne], 24 | 2013, mis en ligne le 17 avril 2014, consulté le 07 juillet 2020. URL : http://journals.openedition.org/paleo/2624 ; DOI : https://doi.org/10.4000/paleo. 2624

Ce document a été généré automatiquement le 7 juillet 2020

\section{c) () $९$}

PALEO est mis à disposition selon les termes de la licence Creative Commons Attribution - Pas d'Utilisation Commerciale - Pas de Modification 4.0 International. 


\section{Art mobilier inédit du gisement de Bourrouilla à Arancou (Pyrénées-Atlantiques, France) : données techno-stylistiques et chrono-culturelles}

Unpublished mobile art from Bourrouilla cave in Arancou (Pyrénées-

Atlantiques, France) : techno-stylistic and chronocultural data

Lise Aurière, François-Xavier Chauvière, Frédéric Plassard, Carole Fritz et Morgane Dachary

Nous remercions Anne Eastham, Sandrine Costamagno, Stéphane Madelaine et Clément Birouste pour la détermination anatomique et spécifique des pièces osseuses ornées. Plusieurs pièces présentées dans l'article ont fait l'objet de restaurations réalisées par les sociétés Materia Viva, ArtéMuse et Utica. Ces restaurations ont été financées par le SRA Aquitaine et le Musée National de Préhistoire des Eyzies que nous remercions sincèrement. Nous remercions aussi Jean-Jacques Cleyet-Merle, directeur du MNP, ainsi que Peggy Bonnet-Jacquement et Bernard Nicolas pour nous avoir facilité l'accès au matériel désormais conservé au MNP. Le site de Bourrouilla est propriété de la commune d'Arancou qui apporte son soutien matériel à la réalisation des fouilles ; qu'elle trouve ici l'expression de nos remerciements. Merci encore à Christine DesdemainesHugon, pour son aide dans la rédaction du résumé et des légendes en anglais.

\section{Introduction}

\section{Localisation et présentation du site}

Bourrouilla est une petite grotte située dans les Pyrénées-Atlantiques, sur la commune d'Arancou. Elle se trouve distante de $9 \mathrm{~km}$ des abris de la falaise du Pastou (Duruthy, Grand Pastou, Petit Pastou et Dufaure) et de $15 \mathrm{~km}$ de l'ensemble Isturitz-Oxocelhaya- 
Erberua (fig. 1). La découverte du gisement a eu lieu en 1986, en même temps qu'une fouille clandestine qui affectait l'intérieur de la cavité. Après le tamisage et le tri des déblais de la fouille clandestine mettant en avant le potentiel et la richesse du site, puis la réalisation d'un sondage extérieur visant à cerner l'extension des occupations et la puissance de la stratigraphie (Chauchat et al. 1999), des fouilles programmées ont été entreprises dès 1998, d'abord sous la responsabilité de Cl. Chauchat, puis sous celle de M. Dachary.

2 Le gisement est classiquement subdivisé en quatre secteurs (fig.2) : la zone extérieure (bandes 25 à 28), le vestibule à l'aplomb du porche (bandes 22 à 24 ), le couloir (bandes 19 à 21) et la salle du fond (bandes 15 à 18). Les occupations magdaléniennes sont documentées par les fouilles conduites dans le secteur extérieur, dans le vestibule et dans la salle du fond.

Bien que le remplissage connaisse des variations latérales, la stratigraphie est identique dans le vestibule et la zone extérieure (Dachary 2005) : sous des niveaux d'occupations holocènes - Age du Fer, Néolithique et Mésolithique (Dachary et al. 2013) - se trouvent les niveaux pléistocènes. L'ensemble $\mathrm{A}$, attribuable au Magdalénien terminal, correspond aux dernières installations au cours du Tardiglaciaire, peu documentées dans le secteur (Dachary et al. 2008 et sous presse). Les occupations du Magdalénien supérieur sont réunies sous la dénomination d'ensemble B. Il s'agit d'une série d'occupations ayant conduit à l'identification de 3 phases majeures (Chauchat et al. 1999) sur la base de l'analyse de la répartition verticale du matériel. Assez homogène, celui-ci se caractérise par la présence de harpons à deux rangs de barbelures, d'un outillage lithique riche en burins et en lamelles à dos et d'une pointe à cran (Bonnissent et Chauvière in Chauchat et al. 1999; Dachary in Chauchat et al. 1999). Outre la fabrication sur place de lamelles aux dépens de nucléus sur tranches d'éclats, sur blocs et, en sommet de séquence, sur nucléus de type grattoirs carénés, les lames sont majoritaires parmi les supports transformés (Dachary 2002). La faune est dominée par le Cerf qu'accompagnent le Cheval et le Renne (Fosse in Chauchat et al. 1999).

Sous l'ensemble $\mathrm{B}$, l'ensemble $\mathrm{C}$, fouillé sur une surface restreinte, est difficile à caractériser bien qu'il ait fourni des pointes à base fourchue et un outillage sur lamelles où abondent les troncatures (Bonnissent et Chauvière in Chauchat et al. 1999 ; Dachary in Chauchat et al. 1999). Il pourrait se rapporter à la période charnière entre Magdalénien moyen et Magdalénien supérieur (Pétillon 2007). 
Figure 1 - Carte de localisation du site de Bourrouilla à Arancou (Pyrénées-Atlantiques, France).

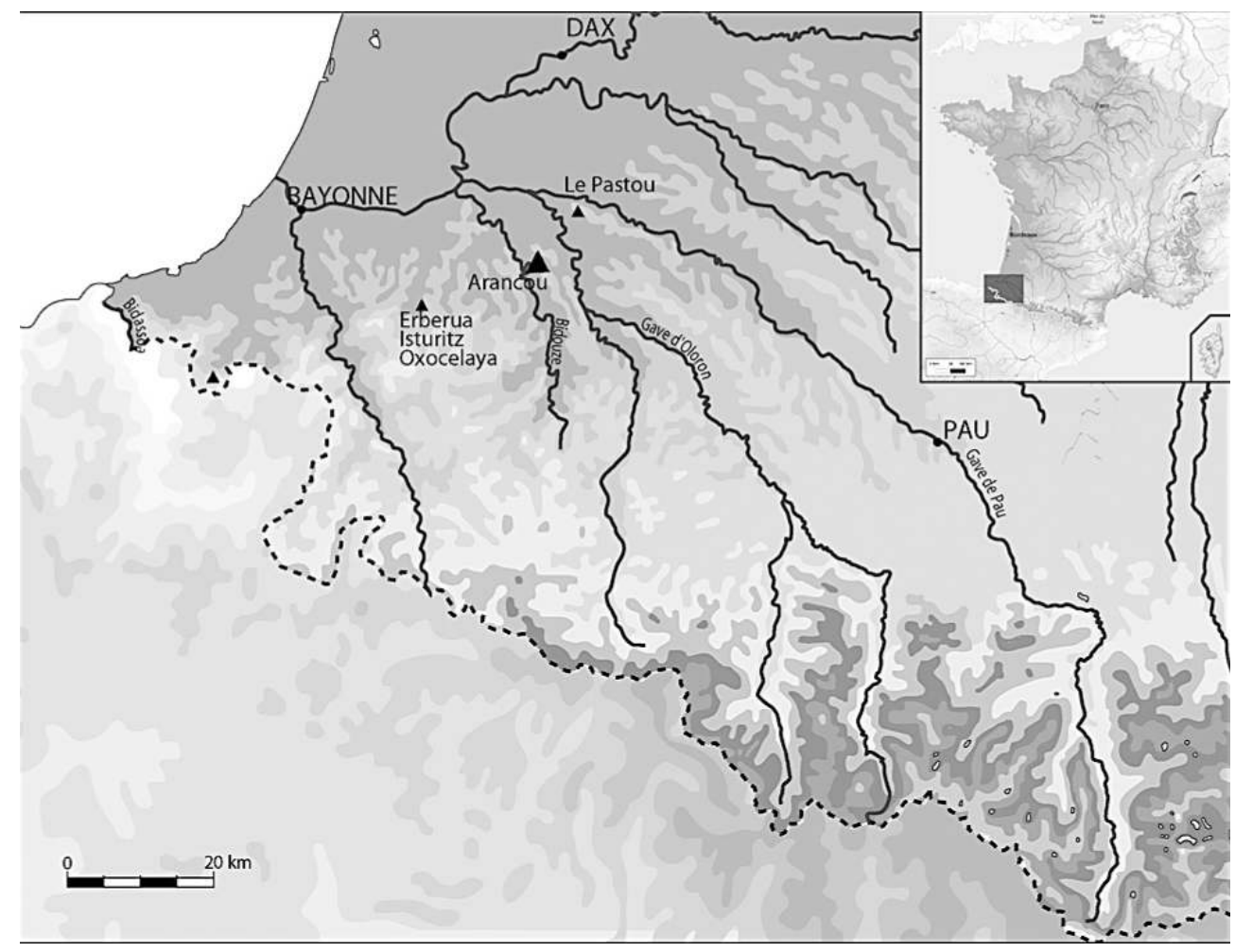

5 L'US 2007, fouillée dans la salle du fond, se présente jusqu'ici comme une banquette en bordure de la fouille clandestine ; elle a été fouillée uniquement sur une surface de $3 \mathrm{~m}^{2}$ environ. A ce jour, aucun lien physique (remontage, raccord entre vestiges, raccord stratigraphique) n'a pu être établi entre la salle du fond et le vestibule. D'abord décrite en coupe comme un ensemble homogène de $70 \mathrm{~cm}$ d'épaisseur, l'US 2007 s'est révélée à la fouille constituée de plusieurs niveaux caillouteux d'une dizaine de centimètres d'épaisseur moyenne, peut-être lenticulaires, de couleur sombre et très riches en matériel archéologique. Les différents niveaux sont séparés par de minces couches de limon jaune stérile. On distingue ainsi l'US 2007 sensu lato et, de haut en bas, les US 2007A, 2007B, 2007CC, et 2007E.

Archéologiquement, les différents niveaux sont similaires (Dachary et al. 2008). La grande faune est dominée par le Cerf et le Renne, qu'accompagnent une avifaune (chouette Harfang pour l'essentiel) remarquablement abondante et bien conservée, ainsi que des vestiges d'ichtyofaune. L'outillage sur lamelles domine l'industrie lithique, bien que les burins soient abondants. L'industrie osseuse associe travail du bois de Cervidé (harpons, pointes de projectiles et pièces techniques) et travail de l'os (aiguille à chas). La parure est représentée par des fragments de coquillages, des incisives d'herbivores sciées et des craches de Cerf percées.

D'un niveau à l'autre, quelques différences sont perceptibles dans la faune (abondance relative de l'avifaune et de l'ichtyofaune), comme dans l'industrie lithique (abondance variable des lamelles). En outre, le secteur fouillé correspond pour l'US 2007AB à une structure de combustion et à une zone de rejet tandis que, pour l'US 2007E, le carré L17 est clairement une zone de rejet. 
Figure 2 - Plan du site de Bourrouilla avec localisation des secteurs fouillés.

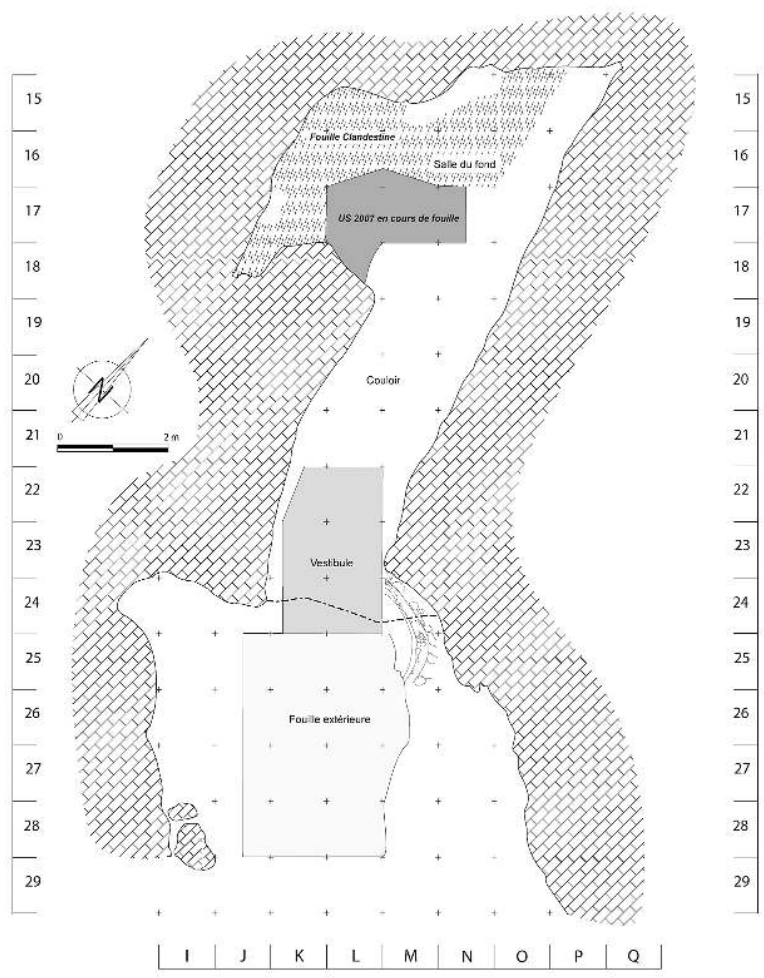

8 Au contact de l'US 2007, et n'apparaissant que sous forme de lentilles, l'US 2006 se compose de sédiments remobilisés depuis le vestibule et contenant un matériel archéologique trié attribuable au Magdalénien supérieur.

9 Les relations chronologiques entre les différents niveaux de l'ensemble B et de l'US 2007 sont difficiles à établir bien que ces niveaux correspondent sans doute à des occupations répétées sur une période courte, au cours du Magdalénien supérieur (tabl. 1). 
Tableau 1 - Datations radiocarbone obtenues sur les niveaux magdaléniens de Bourrouilla d'après Fontugne et Hatté in Chauchat et al. 1999 et Szmidt et al. 2009. Les dates sont calibrées avec le logiciel calib 6.0.1 (Stuiver et Reimer 1993) et exprimées à 2 sigma.

\begin{tabular}{|l|c|c|c|c|}
\hline \multicolumn{1}{|c|}{ Couche } & Attribution culturelle & Référence & Age BP & Age cal BP \\
\hline Couche B2 & Magdalénien supérieur & $\begin{array}{c}\text { GIF 9986 } \\
\text { (LSM 9986) }\end{array}$ & $12395 \pm 35$ & $14920-14110$ \\
\hline Couche B2 & Magdalénien supérieur & GIF 10002 & $12260 \pm 120$ & $14900-13840$ \\
\hline Couche B3 & Magdalénien supérieur & GIF 10255 & $12780 \pm 40$ & $15590-14910$ \\
\hline Couche C1 & Magdalénien moyen & GIF 10234 & $12700 \pm 40$ & $15260-14680$ \\
\hline Couche C2 & Magdalénien moyen & GIF 10254 & $13220 \pm 80$ & $16690-15390$ \\
\hline Couche C4 & Magdalénien moyen & GIF 10235 & $12710 \pm 90$ & $15610-14550$ \\
\hline US 2007, Harfang & Magdalénien supérieur & OxA-19696 & $12540 \pm 50$ & $15120-14410$ \\
\hline US 2007, Renne & Magdalénien supérieur & OxA-19698 & $12610 \pm 55$ & $15210-14500$ \\
\hline US 2007, Cerf & Magdalénien supérieur & OxA-19697 & $12880 \pm 50$ & $15930-15000$ \\
\hline
\end{tabular}

\section{Matériel d'étude et problématique}

Lors du tamisage des déblais de la fouille clandestine, 22 fragments d'objets d'art mobilier ont été découverts, dont la moitié porte des représentations figuratives. Ces pièces ont déjà fait l'objet d'études très détaillées, que ce soit d'un point de vue stylistique ou technologique (Fritz et Roussot in Chauchat et al. 1999 ; Fritz 1999). Il s'agit notamment de 13 objets réalisés sur hémi-côte : lissoirs et pendeloques à bélière. Les faces supérieures (tissu compact) présentent des traces de raclage et/ou polissage, tandis que le tissu spongieux de la face inférieure témoigne de différentes étapes de régularisation. Pour six pièces, les bords ont été aménagés de festons. Le décor est souvent très détaillé et de très grande qualité d'exécution (ibid.). Les déblais ont également livré des fragments de baguette demi-ronde à décor géométrique, et un tube en os d'oiseau portant deux représentations de chevaux et d'oiseau.

11 Aussi, depuis le début des fouilles programmées en 1998, les découvertes d'éléments d'art mobilier sont-elles très attendues; elles constituent l'un des enjeux majeurs des recherches menées dans la grotte de Bourrouilla à Arancou. Jusqu'à récemment, la plupart des vestiges rapportables à cette catégorie de la culture matérielle provenaient de zones remaniées du site (fouille clandestine, US 2006, US 2017). La mise au jour, depuis 2009, d'artefacts recueillis en stratigraphie ainsi que le raccord physique entre l'une de ces pièces et d'autres au contexte moins assuré, sont par conséquent du plus grand intérêt pour jeter les bases d'une chronologie fine de la production d'art mobilier d'Arancou. Il est désormais possible de proposer une attribution chronoculturelle pour les pièces inédites et pour certains objets issus des déblais de la fouille clandestine. Plus largement, les travaux conduits offrent l'opportunité de reconstituer le contexte archéologique associé aux œuvres d'art découvertes dans la fouille clandestine et de proposer une attribution chronologique pour des objets similaires dans des sites fouillés anciennement et dont la stratigraphie est mal établie.

12 Au-delà des données thématiques, stylistiques et techniques qui sont immédiatement accessibles, et que nous présenterons tout d'abord dans cet article, ce sont également 
les modalités d'accumulation des objets décorés sur matières osseuses ou lithiques dans le site d'Arancou que nous tentons de cerner. Au travers de la présence ou l'absence des marqueurs d'activités que sont les déchets de fabrication, nous cherchons notamment à distinguer les éléments qui ont été fabriqués sur place de ceux qui ont pu être introduits à l'état d'objets finis, ou qui ont pu être emportés hors du site.

L'analyse de la distribution spatiale de ces vestiges spectaculaires offre en outre l'occasion, d'une part, d'identifier la nature des activités en lien avec cet art mobilier, et, d'autre part, de comprendre les relations fonctionnelles que peuvent entretenir ces mêmes activités entre elles, en regard du découpage tripartite de la cavité (zone extérieure, vestibule et salle du fond). Il devient dès lors pertinent d'intégrer l'étude de l'art mobilier à la problématique plus générale de la structuration interne de l'espace habité paléolithique d'Arancou, et de confirmer les premiers indices d'une différenciation entre zones de fabrication, aires d'utilisation ou de rejet entrevus à l'issue de l'analyse d'autres types de vestiges (Dachary 2005 ; Dachary et al. 2008).

En définitive, la mise en relation contextuelle des différentes données relatives à l'art mobilier s'inscrit dans l'approche plus large des continuités/discontinuités technologiques qui sous-tendent la formation des assemblages archéologiques d'Arancou. Elle conditionne également l'appréhension de la variabilité d'une expression graphique très particulière sur supports mobiles, tant à l'échelle du site qu'à celle du Magdalénien des Pyrénées occidentales (Dachary et al. 2006).

\section{1 - Méthodologie}

En complément de l'approche thématique et stylistique, nous avons opté pour une analyse résolument technique des éléments d'art mobilier d'Arancou. Le concept de chaîne opératoire (Leroi-Gourhan 1943 ; Pelegrin et al. 1988; Perlès 1991) est l'outil méthodologique qui a permis de sérier, dans un ordre de succession stricte, l'ensemble des traces observées.

Les stigmates répertoriés sont en premier lieu relatifs aux différentes phases techniques qui ont régi la fabrication des objets décorés, et essentiellement à la phase de façonnage proprement dite (préparation des surfaces à graver par raclage et/ou polissage ; mise en place de l'ornementation par incisions fines ou profondes, etc.). D'autres indices tels que les cassures ou les traces de chauffe ont offert l'occasion de s'interroger sur les modalités d'utilisation et d'abandon des objets d'art mobilier.

Tous les objets ont été observés à l'échelle macroscopique et sous différents grossissements (jusqu'à 20 fois) à l'aide d'une loupe binoculaire classique (Olympus, grossissement x10) et d'une loupe (Dinolite) permettant l'observation sur l'écran d'ordinateur.

\section{2 - Présentation des nouvelles découvertes (tabl. 2)}


Tableau 2 - Inventaire des objets décrits dans l'article.

\begin{tabular}{|c|c|c|c|c|c|c|c|c|c|}
\hline Anneb & Carre & \begin{tabular}{|c|}
$n^{*}$ inventaire \\
\end{tabular} & Localisation & Stratigraphie & Materiau & $\begin{array}{c}\text { Dimensions } \\
\text { (Len mm) }\end{array}$ & $\begin{array}{l}\text { Próparation du } \\
\text { suupoort }\end{array}$ & Decor & Illustration \\
\hline ARA03 & k24 & 2170 & \begin{tabular}{|l|l|} 
vestibule-extérieur \\
\end{tabular} & Ensemble B & fémur droit, Cert & $86 \times 24 \times 6$ & 1 & gravure, figuration nageoire caudale de mammilère marin & fig. 3 \\
\hline ARA11 & L23 S1 & $300 / 302$ & 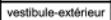 & Ensemble $\mathbf{B}$ & gres brenn-rovge & \multirow[b]{2}{*}{$10 \times 2.5$} & 7 & gravures fines, patte & 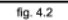 \\
\hline ARA11 & L23S1 & $300 / 302$ & \begin{tabular}{|l|} 
vestibule-extérieur \\
\end{tabular} & Ensemble B & grès brun-rouge & & 1 & gravures fines, patte & fig. 4.2 \\
\hline$\widehat{A R A 10}$ & $\angle 24$ & 2937 & vestibule-extérieur & B2 & grès brun-rouge & $38 \times 28 \times 3$ & 7 & gravures fines, tête indótereminée & fig. 4.1 \\
\hline ARA10 & L24 & 3117 & vessibulie-exterieur & Ensemble B & gress brun-rovge & $24 \times 24 \times 3$ & 1 & gravures fines, patte & fig. 4.2 \\
\hline ARAO9 & $\angle 24$ & 2838 & vestibule-extérieur & Ensemble $B$ & grès brun-rouge & $33 \times 28 \times 7$ & 1 & gravures fines & \\
\hline ARA90 & k25 & 507 & 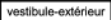 & 82 & plaquette de gres & $77 \times 7210$ & 1 & Incisions non figuratives? & \\
\hline ARA90 & 125 & 199 & 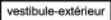 & 82 & plaquette de gres & $93 \times 72 \times 17$ & 1 & incisions non figuratives? & \\
\hline ARA10 & 124 & 3230 & 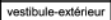 & 82 & roche metamorphique & $68 \times 41$ & $T$ & fraces couleur rouge & \\
\hline ARA09 & L16 & 110 & intérieur grotte & US2007 & 2ème cote gauche, Corf & $137 \times 18 \times 4$ & 1 & gravure, figuration de deux canildés & fig. 6 \\
\hline ARA06 & & & Interieur grotte & US 2003 & bois de cervidé & $28 \times 6 \times 5$ & $T$ & gravures, motfls en "parenthésese" & $\begin{array}{l}\text { fig. } 15.4 \\
\text {. }\end{array}$ \\
\hline ARA 98 & & & intiéneur grotte & Foulle clandestine & bois de cervidé & $74 \times 11 \times 4$ & 1 & baguette demi-ronde, motts en "parentheseses" & fig. 15.2 \\
\hline RH18A01-465-ATR| & & & inténieur grotte & \begin{tabular}{|l|} 
Fouille clandestine \\
\end{tabular} & bois de cervidé & $18 \times 8 \times 3$ & $t$ & gravures, motffs en chevirons & fig. 15.3 \\
\hline ARA11 & 117 & 3092 & intéreueur grotete & US2007 & Cote? & $20 \times 11 \times 2$ & raclage & gravure, figuration dun cervidé & fig.7 \\
\hline ARA11 & L17 & 2777 & Intiéreuc grotate & US2007 & Cotte, cerf & $103 \times 17 \times 6$ & 1 & gravure, figuration de deux chevaux & fig. 5 \\
\hline \multirow[t]{8}{*}{ ARA09 } & L17 \$1 & 370/372 & intíneueur grotte & US 2007E & hém-octe & $7 \times 7 \times 1$ & raclage & |"ilissoli" à bord festonné & 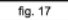 \\
\hline & & & intérieur grotte & \begin{tabular}{|l|} 
Fouille clandestine \\
\end{tabular} & hémi-cote & $22 \times 9 \times 2$ & $\begin{array}{l}\text { raclage, polissage } \\
\end{array}$ & pièce festonnée, figuration d'un salmonidé & fig. 10 \\
\hline & & & Intérieur grotte & 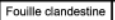 & héml-oste & $32 \times 10 \times 3$ & I & geravures, figuration de deux ours & fig. 11 \\
\hline & & & intitenteur grotate & Fouille dandestine & hemil-oble & $19 \times 5 \times 2$ & 1 & gravure, figuration d'un cenvide & fig. 12 \\
\hline & & & interieur grotte & \begin{tabular}{|l|} 
Fouille clandestine \\
\end{tabular} & hémi-cote & $41 \times 14 \times 1$ & radlage & gravure, motifs en triangle & fig. 13 \\
\hline & & & intérieur grotte & \begin{tabular}{|l|l} 
Fouille clandestine \\
\end{tabular} & hémi-cote & & raclage, polissage & pendeloque à bêlïre, gravure & fig. 14 \\
\hline & & & intiténeur grotute & Fouille clandestine & hémi-cote & $31 \times 10 \times 1$ & raclage & Flissoir à bord festonné & fig. 17 \\
\hline & & & intúneutr grotate & 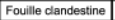 & hém-cote & $19 \times 10 \times 1$ & raclage & |"lissoir" à bord festonné & fig. 17 \\
\hline \multirow[t]{3}{*}{ ARA05 } & 417 & 1074 & intérieur grotte & US2007 & Una, Chouette harfang & $80 \times 5$ & raclage & gravure, non figuratiff & fig. 8 \\
\hline & & & intérieur grotte & \begin{tabular}{|l|} 
Fouille clandestine \\
\end{tabular} & bois de cervidé & $47 \times 12 \times 5$ & 7 & baguette demi-ronde à tubérositếs & fig. 15.1 \\
\hline & & & Intieneur grothe & Fouille dandestine & calcarre & $63 \times 34 \times 9$ & 1 & gravures fines & fig. 16.1 \\
\hline ARA11 & L17 & 3122 & intérieur grotte & US 2007 & calcaire allochtone & $45 \times 32 \times 14$ & 1 & gravure fine & \\
\hline ARA12 & N17 S1 & $376 / 378$ & intérieur groctte & US 2007 & calcaire allochtone & $33 \times 16 \times 4$ & 1 & gravure profonde & fig. 9.1 \\
\hline ARA04 & M17 & 856 & intínéneur grotte & US 2007 & calcaire local & $70 \times 70 \times 7$ & 1 & gravures fines & \\
\hline ARA05 & N17 & 454 & intínieur grotte & US 2007 & calcaire local & $67 \times 50 \times 10$ & 1 & gravures fines & \\
\hline ARA05 & N17 & 464 & inténeur grotte & US 2007 & calcaire local & $48 \times 33 \times 6$ & 1 & gravures fines & \\
\hline ARA04 & M16 S3 & 348/350 & intéreuer grotute & US 2006 & galet & $15 \times 12 \times 7$ & 1 & gravure & \\
\hline \multirow[t]{3}{*}{ ARA07 } & & & inténeur grotte & US 2017 & grìs & $75 \times 38$ & 1 & gravures fines, tracess couleur rouge & fig. 16.2 \\
\hline & & & intenieur grotte & Fouille clandestine & gré & $149 \times 83 \times 16$ & 1 & Incisions non figuratives? & \\
\hline & & & intérieur grotte & Fouille clandestine & grís & $135 \times 90 \times 15$ & 1 & lincisions non figuratives ? & \\
\hline ARAO9 & L17 & 2363 & interieneur grotte & US 2007 & gràss àgrain grossier & $59 \times 47 \times 6$ & 1 & gravure & \\
\hline ARAO9 & L17 & 2435 & interieueur grotte & US 2007 & grass à grain grossier & $95 \times 66 \times 8$ & 7 & gravure & \\
\hline ARA05 & M17 & 1223 & intírieur grotte & US 2007 & grisia a grain n rossier & $85 \times 55$ & 1 & gravurre, arrierse-train de boviné & fig.9.2 $92>0$ \\
\hline
\end{tabular}

2.1 - Les pièces inédites des niveaux en place dans le vestibule et à l'extérieur (ensemble B)

2.1.1 - Fragment de diaphyse (ARA03 K24 2170) (fig. 3) 
Figure 3 - Diaphyse de fémur de Cerf gravée (pinnipède ?). Photos F. Plassard, croquis de lecture L. Aurière.

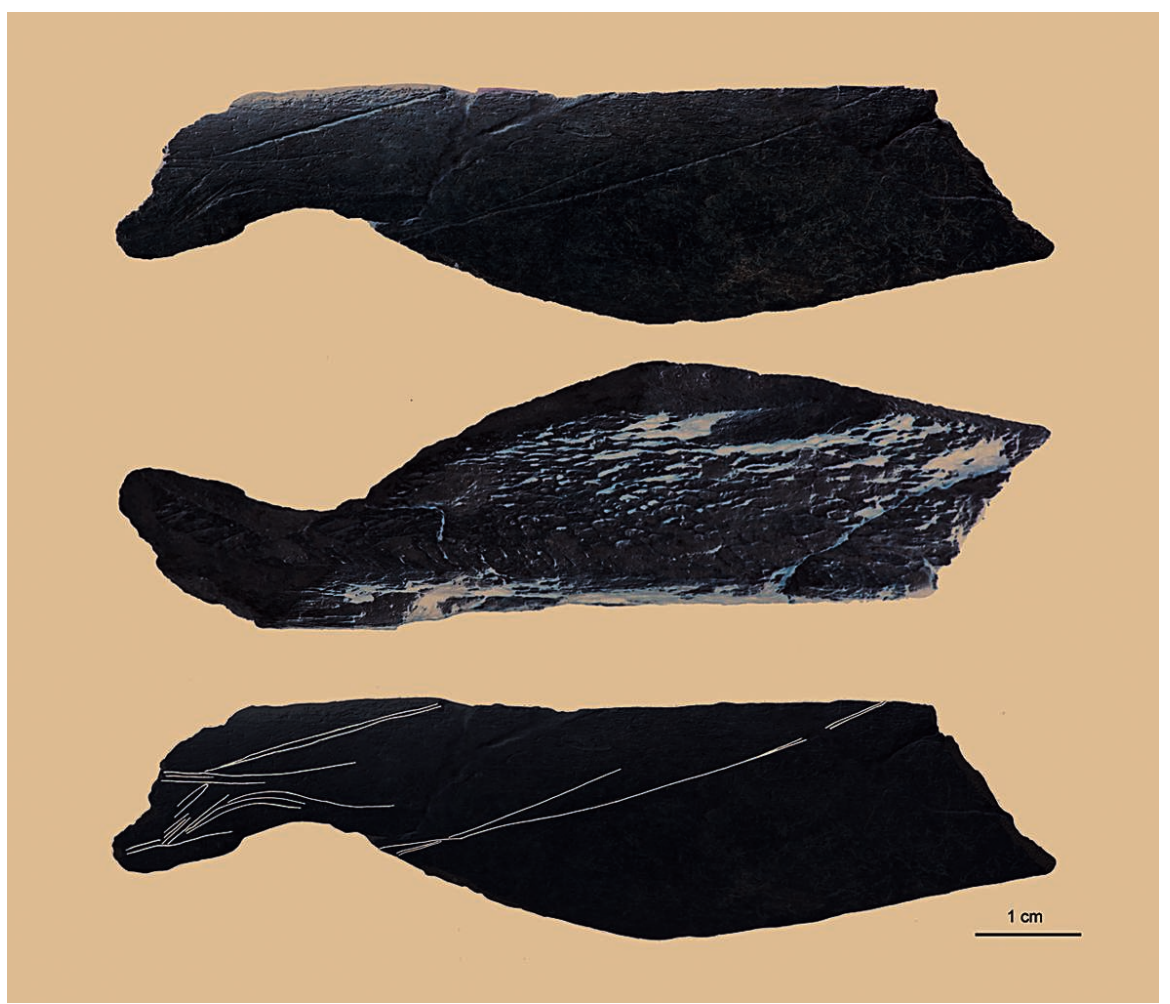

Matière : fragment de fémur droit de Cerf

Dim : 8,6 × 2,4 x 0,6 cm

Section : Hémi-circulaire

Contour : Bords cassés

Profil : Rectiligne

Il s'agit d'un fragment de diaphyse cassé anciennement sur tous ses bords, postérieurement à la gravure. La surface ne présente pas de stigmates clairs de préparation préalable au décor. Celui-ci est constitué de tracés assez larges obtenus par plusieurs passages. Le motif incomplet est difficilement interprétable. Toutefois, la représentation de la nageoire caudale d'un pinnipède pourrait être proposée. Cette lecture s'accorderait avec la morphologie fuselée du corps. Par ailleurs, les mammifères marins ne sont pas rares dans l'art magdalénien des Pyrénées occidentales et des Cantabres (cétacé à Arancou (fig. 18.6), phoque à Duruthy, ..., Serangeli 2003).

\subsection{2 - Pièces sur support minéral}

Dix pièces possiblement ornées sur support minéral sont recensées pour le Magdalénien supérieur à l'extérieur (Sondage KL 25-28) et dans le vestibule (KL 23-24). Deux plaquettes de grès (ARA90 K25 507 et ARA90 L25 199) portent des traits dont le caractère délibéré et symbolique est difficile à vérifier. Un fragment de roche métamorphique (ARA10 L24 3230) présente des traces d'ocre. Rien ne permet d'affirmer qu'il s'agit d'une pièce ornée plutôt que d'une coloration accidentelle ou consécutive à une action technique.

21 En revanche, sept pièces en grès brun-rouge, lité, à grain très fin portent des gravures incontestables. Ces vestiges de très petites dimensions proviennent sans doute d'un 
même support aujourd'hui fragmenté. Découvertes dans les carré L23 et L24, certaines de ces pièces se raccordent tandis que d'autres ne peuvent être que rapprochées sur la base du matériau. Trois fragments n'apportent aucune information particulière. En revanche, un autre porte des gravures nettes parmi lesquelles on peut lire une tête non déterminable (fig. 4.1). En profil gauche, on y voit un museau allongé dont l'extrémité est arrondie. L'image est interrompue à droite par le bord cassé du fragment. Les trois derniers fragments raccordent en une petite plaquette sur laquelle on peut lire une patte finement gravée. La détermination spécifique n'est pas possible (fig. 4.2).

Figure 4 - 1- Plaquette de grès gravée de divers traits parmi lesquels une tête animale en profil gauche. 2- Plaquette de grès gravée d'une patte. Photos et croquis de lecture F. Plassard.
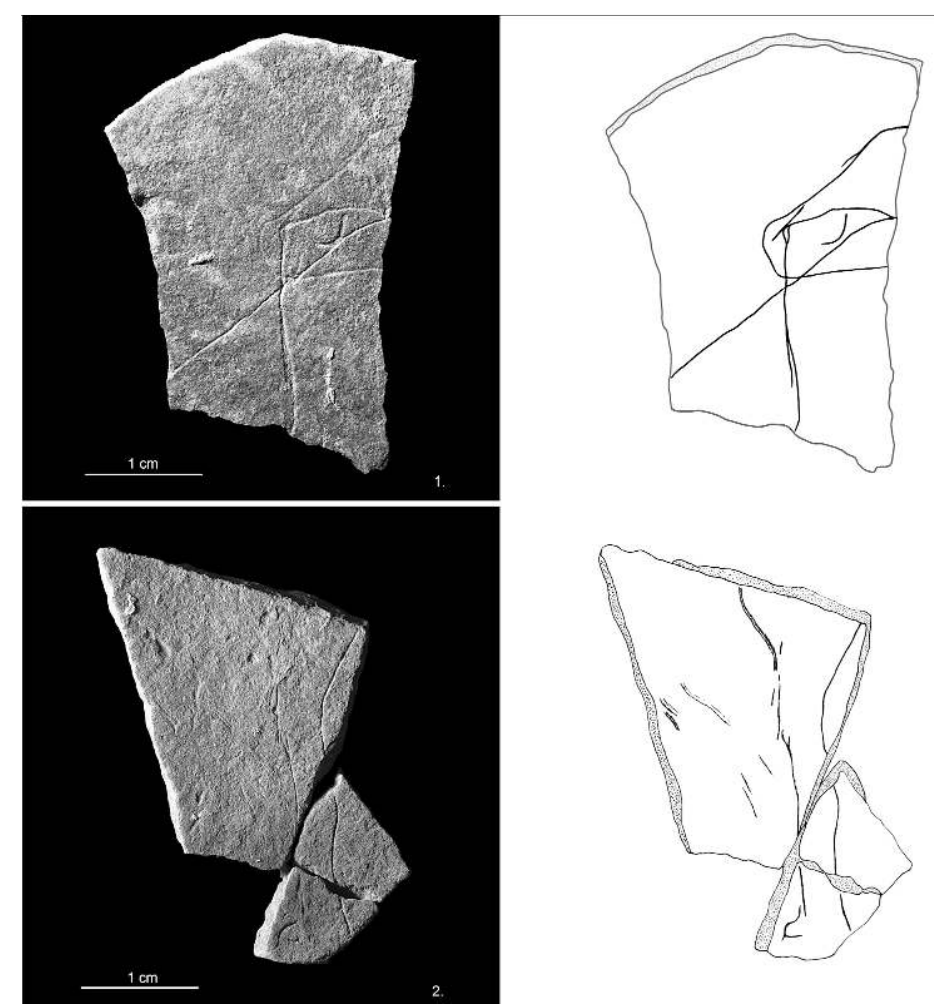

\section{2 - Pièces inédites des niveaux en place dans la grotte (US 2007)}

\subsection{1 - La côte aux chevaux (ARA1 1 L17 2777) (fig. 5)}


Figure 5 - 1- Faces supérieure et inférieure de la côte aux chevaux. 2- Détail de l'arrière-train du sujet de droite (x 20). 3- Détail de la tête du sujet de droite (x 20). 4- Détail de la tête du sujet de gauche ( $x$ 20). 5 - Croquis de lecture. Photos F. Plassard et $L$. Aurière, croquis de lecture $L$. Aurière.

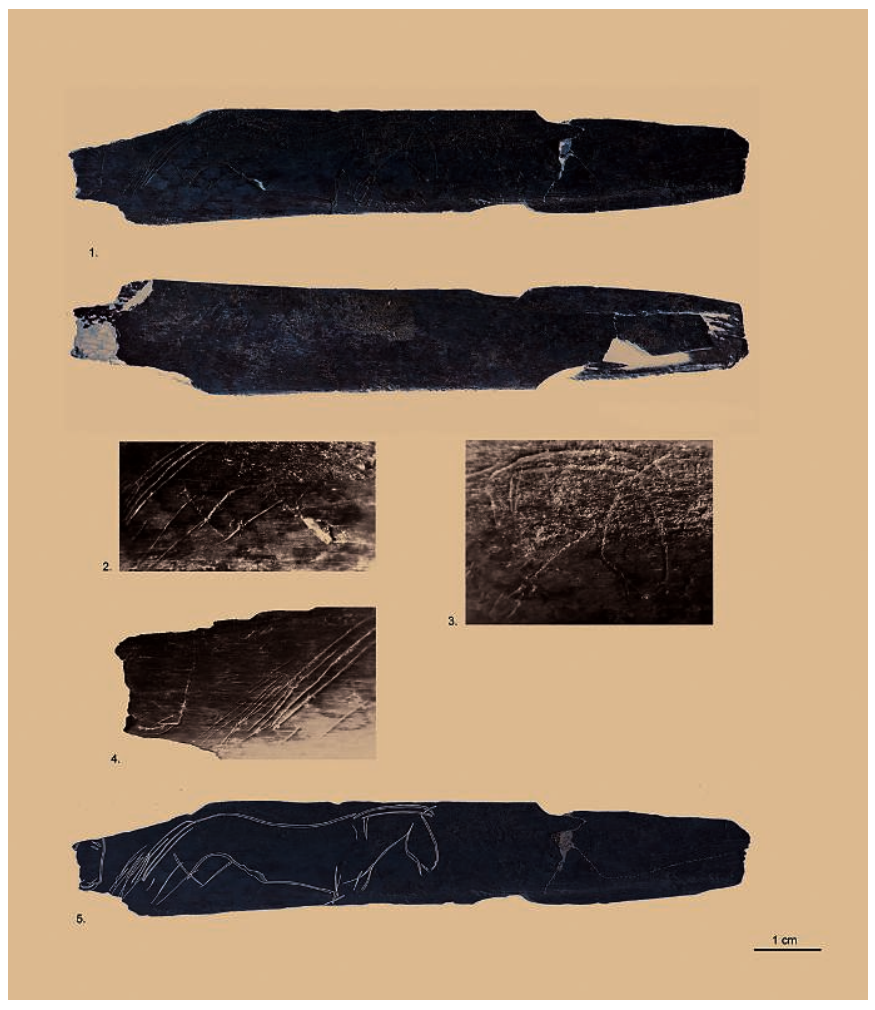

Matière : Os, Côte de Cerf

Dim : 10,3 x 1,7 x 0,7 cm

Section : Plano-convexe

Contour : Convergent vers une des extrémités

Profil : Rectiligne

Par comparaison avec la série des petits lissoirs, cet objet n'offre pas un parfait état de conservation ; cassure, desquamation des surfaces et concrétionnement compliquent la lecture du décor.

Une zone de raclage appuyé est identifiable sur chaque bord, sur une longueur de $1 \mathrm{~cm}$ environ. Il pourrait s'agir des stigmates d'une tentative avortée de bipartition de la côte. Aucune des faces ne porte de traces visibles de raclage préalable à la gravure.

La partie gauche (en l'état actuel de conservation) de la face externe, convexe, est ornée de deux représentations de chevaux en profil droit. La première est limitée à la partie avant de la tête en raison d'une cassure, tandis que la seconde est complète.

Le premier cheval (fig. 5.4) est donc réduit à la représentation du front, du chanfrein, de la lèvre supérieure, de l'œil et d'une partie de la branche montante de la mandibule. Le naseau n'est pas indiqué. L'œil est formé de deux petits traits courbes parallèles, convexes vers le haut. Le chanfrein est rectiligne tandis que le front est légèrement convexe à la hauteur de l'œil. Le bout du nez et la lèvre supérieure sont indiqués sans que le museau soit figuré.

La seconde figuration de cheval se trouve à droite de la première, également en profil droit. Elle est complète mais la tête est moins détaillée (fig. 5.3). Le chanfrein est convexe ; le naseau et la bouche ne sont pas indiqués tandis que l'auge, la ganache et la 
branche montante de la mandibule forment une seule ligne qui rejoint celle du poitrail. L'œil n'est pas visible, mais la surface de l'os est desquamée à cet endroit de telle sorte qu'il n'est pas possible de dire s'il a été ou non figuré. La crinière et l'encolure sont représentées par deux lignes parallèles qui forment un cimier au-dessus du front. Trois incisions plus fines dessinent quelques poils de crinière retombant sur le garrot. Le dos et la croupe sont dessinés d'une même ligne en contre-courbe. La fesse n'est pas indiquée tandis que plusieurs longs traits évoquent la queue. Les membres postérieurs ne sont pas figurés. Dans sa partie postérieure, la ligne ventrale est fortement incurvée vers le haut tandis qu'elle est presque rectiligne vers l'avant. Elle s'interrompt sur le membre antérieur dont l'évocation est limitée à l'avant-bras.

La réalisation de la figure complète est assez maladroite. Les détails internes ne sont pas figurés. Les pattes avant, l'arrière train et la queue présentent des erreurs de positionnement, ou ne sont pas figurés entièrement.

Des maladresses gestuelles sont bien identifiables : elles caractérisent des difficultés dans la maîtrise de l'inclinaison de la partie active de l'outil à la surface de l'os (Fritz 1999). Il s'agit notamment de stigmates d'accident comme celui visible au niveau du ventre, qui a entraîné un arrachement important de matière sur le bord du trait (fig. 5.2). Nous pouvons également noter des sorties de traits, au niveau de la ganache ou encore sur le poitrail.

\subsection{2 - La côte aux canidés (ARA09 L16 110) (fig. 6)}

Figure 6 - 1- Faces inférieure et supérieure de la côte aux canidés. 2- Croquis de lecture. 3- Détail du sujet de gauche (x 30). 4- Détail du sujet de droite (x 30). Photos F. Plassard et L. Aurière, croquis de lecture $\mathrm{L}$. Aurière.

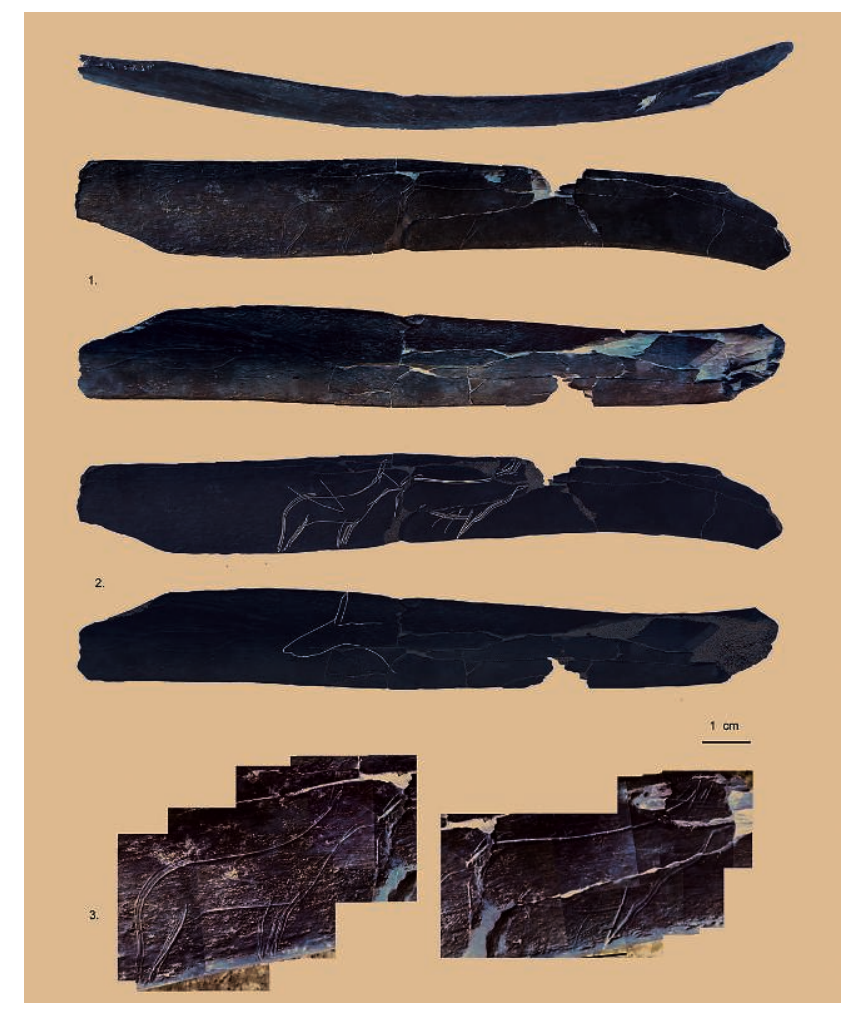

Détermination : Os, 2ème côte gauche de Cerf

$\operatorname{Dim}: 13,7 \mathrm{x} 1,8 \mathrm{x} 0,4 \mathrm{~cm}$ 
Section : Plane

Contour : Bords convergents aux deux extrémités

Profil : Courbe

31 Cette pièce, partiellement brûlée, découverte en 34 fragments, présente un état de conservation moyen rendant difficile la lecture de certains détails. Des stries de raclage assez profondes sont visibles le long d'un des bords, ainsi qu'en partie gauche de la face supérieure.

Sur la face inférieure ont été gravées deux représentations animales en profil droit. Les deux figurations sont complètes mais endommagées par les cassures du support. Seuls leurs profils sont indiqués. L'ensemble est statique, les membres bien campés sur le bord du support qui semble jouer le rôle d'une ligne de sol. L'animal de gauche est le plus complet. Il présente une tête triangulaire avec un museau pointu et une oreille saillante. La ligne dorsale est presque plate avec un poitrail droit et un cou resserré vers la montée de la tête. L'arrière-train est très arrondi, figuré par un tracé dédoublé. Le membre postérieur est figuré légèrement décalé vers l'arrière et la queue est absente. Deux incisions obliques et convergentes sont positionnées sur le flanc, en partie supérieure. Le second animal est moins lisible car morcelé par de nombreuses cassures. La ligne cervico-dorsale est très légèrement concave, la tête (désormais incomplète) semble plus épaisse, et le poitrail plus massif. La ligne ventrale est vestigiale, le membre antérieur est droit et finit en pointe. Un poitrail massif bien marqué est réalisé dans la continuité de cette dernière. L'ensemble est poursuivi par l'amorce d'un cou massif et large qui semble associé à une amorce de tête triangulaire surmontée de quatre tracés verticaux qui semblent figurer les oreilles.

Tout comme pour le premier sujet, deux incisions obliques et convergentes se trouvent sur le flanc, mais cette fois dans la partie inférieure. La détermination spécifique de ces représentations est délicate. La morphologie et l'attitude des animaux conduisent à hésiter entre cervidés et canidés. Le sujet de gauche nous fait cependant privilégier la dernière hypothèse.

Sur la face supérieure, à gauche du raclage, on peut lire une tête d'animal en profil gauche dont l'exécution est sommaire. Le tracé rectangulaire en partie supérieure s'apparente à la figuration de l'oreille du premier canidé de l'autre face.

La réalisation des figures est très concise, sans détail anatomique, et nous pouvons noter une certaine hésitation dans la réalisation du dessin. Plusieurs sorties de traits sont visibles, notamment dans la réalisation de la courbe du poitrail de l'animal de gauche. Par ailleurs, certains tracés sont peu appuyés, tandis que d'autres présentent des «broutages » interprétables comme une mauvaise inclinaison de l'outil à la surface de l'os (Fritz 1999).

\subsection{3 - La plaquette osseuse au cervidé (ARA11 L17 3092) (fig. 7)}


Figure 7 - Fragment osseux gravé d'une tête de cervidé. Photos F. Plassard, croquis de lecture L. Aurière.

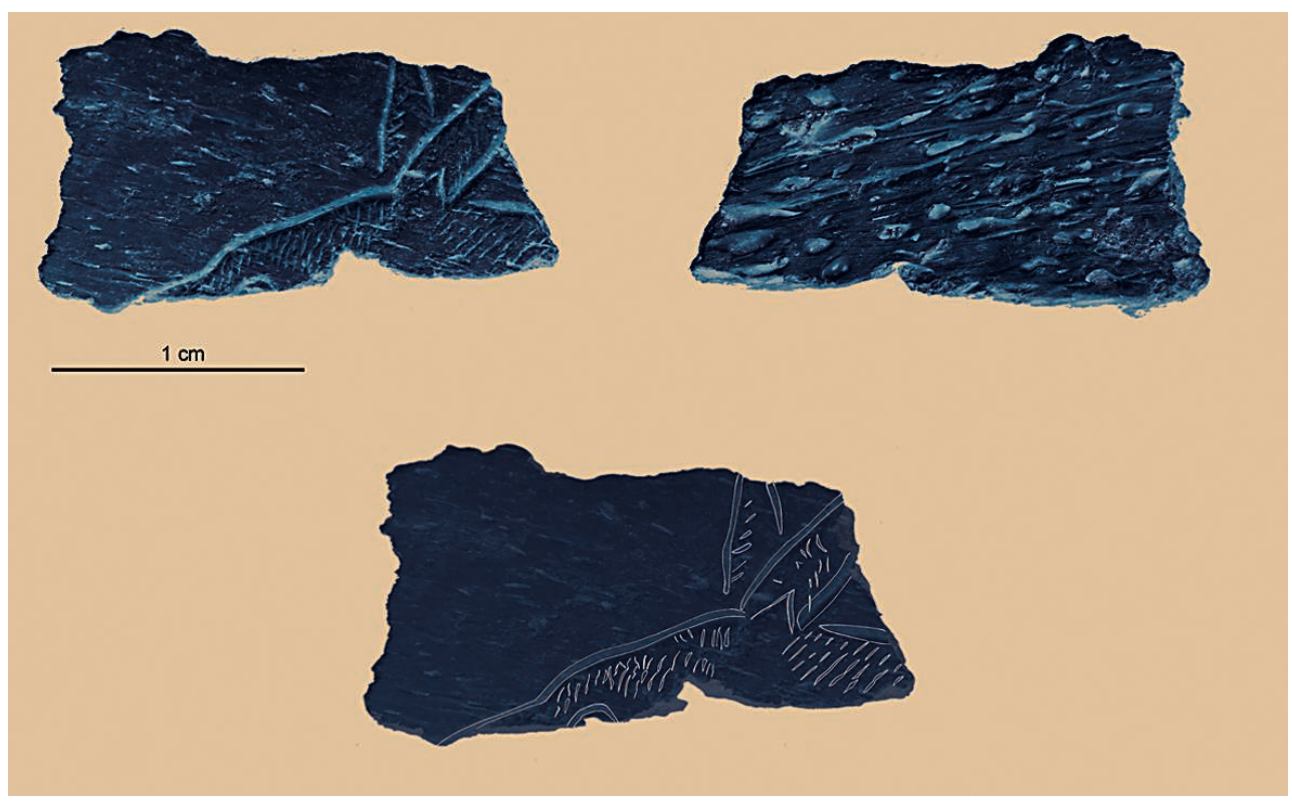

Matière : Os, Côte?

Dim : 2 × $1,1 \times 0,2 \mathrm{~cm}$

Section : Plane

Contours : Bords cassés

Profil : Rectiligne

Il s'agit probablement d'un fragment d'hémi-côte cassé sur tous ses bords, postérieurement à la gravure. La pièce est en très bon état de conservation. L'ensemble de la surface présente de fins stigmates d'un raclage antérieur à la gravure.

Malgré le caractère très fragmentaire de cet objet, un segment de tête en profil gauche est bien visible. Le chanfrein dessine le bourrelet sub-orbitaire avant de s'infléchir jusqu'à la naissance des oreilles. Celles-ci sont grandes et figurées en perspective. L'amorce de l'encolure est aussitôt interrompue par la cassure du support. L'œil à l'état vestigial est visible sur le bord inférieur de la pièce. L'ensemble de la tête est recouvert de tirets courts parallèles ou sub-parallèles qui figurent le pelage de l'animal. La taille des oreilles et la forme du chanfrein orientent l'interprétation vers un cervidé.

Techniquement, la gravure est très sûre, profonde pour les contours et plus légère pour les attributs internes.

\subsection{4 - Os d'oiseau (ARA05 L17 1074) (fig. 8)}


Figure 8 - Ulna de chouette Harfang gravé. Photos F. Plassard.

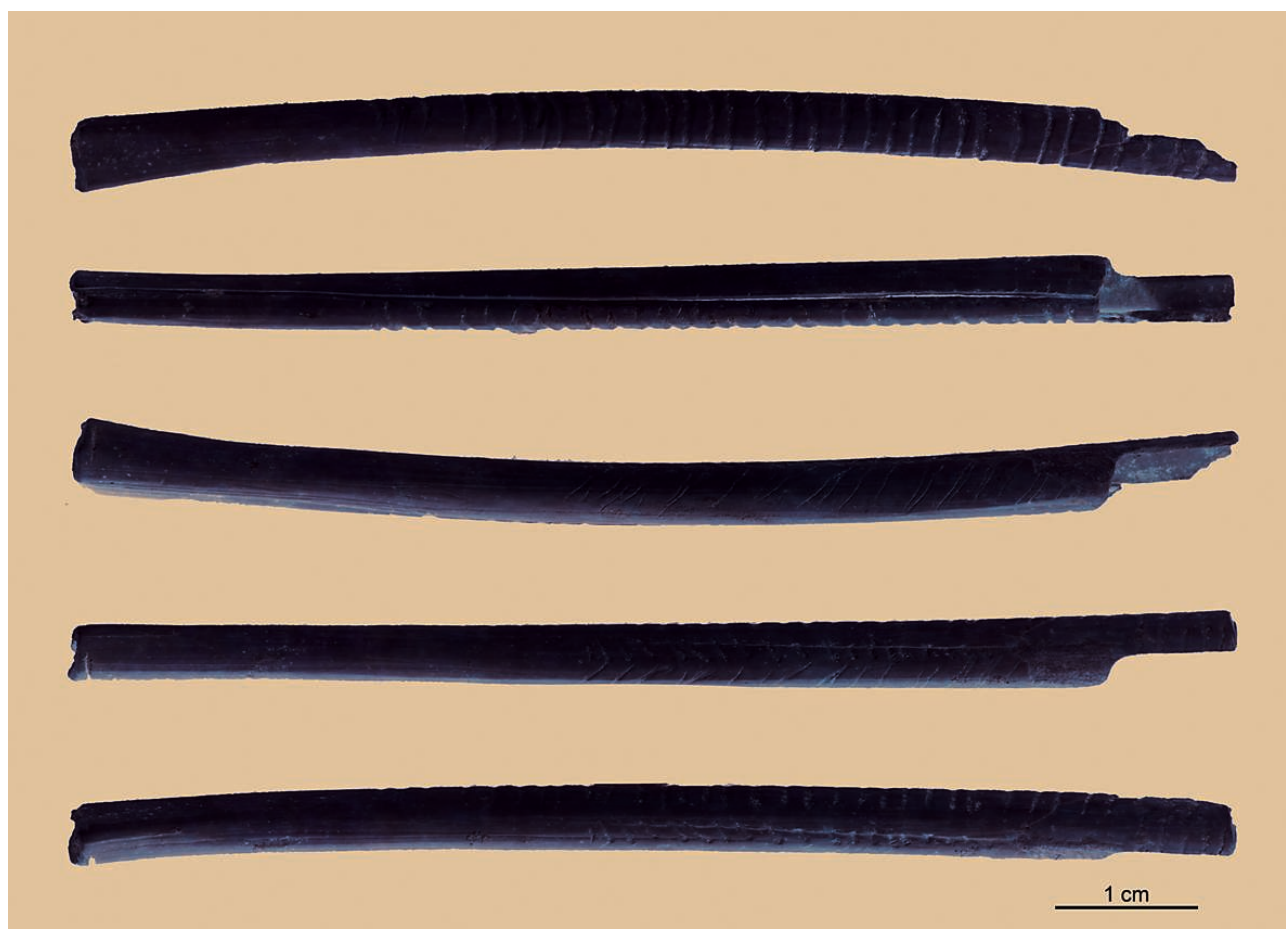

Dim : Long. : $8,05 \mathrm{~cm}$; diam. $\max : 0,5 \mathrm{~cm}$

Section : Circulaire

Contour : Bords parallèles

Profil : Courbe

40 Cet os d'oiseau est cassé à l'une de ses extrémités postérieurement, à la mise en place du décor. Une fissure longitudinale est présente sur sa longueur. Cette fragilité a été initialisée par un rainurage longitudinal.

41 La surface a été raclée dans un premier temps. L'une des extrémités (à gauche sur la photo) porte les traces d'un sciage au trait de silex. Deux séries d'incisions couvrent plus des deux tiers de la longueur de l'objet. La première série se compose d'incisions longues et fines, tandis qu'une seconde est faite de tracés plus courts et assez profonds.

Il semble que les premiers tracés aient été réalisés avant le rainurage, tandis que les seconds ont été mis en place après, en s'intercalant entre les incisions princeps.

\subsection{5 - Pièces sur support minéral}

43 Jusqu'ici, huit pièces gravées sur support minéral ont été découvertes au cours des fouilles des niveaux du Magdalénien supérieur (US 2007) de la grotte. Trois d'entre elles (dont deux remontent) sont des fragments de calcaire identiques à l'encaissant, gravés de lignes très fines, peu nombreuses et sans interprétation. Deux autres sont des fragments de calcaire étrangers au site. L'un est gravé d'un motif allongé réalisé en gravure fine (fig. 9.1) tandis que l'autre est constitué d'une ligne unique profonde. Un petit fragment de galet porte des incisons nettes mais qui ne forment aucun motif figuratif ou géométrique. Enfin trois plaquettes de grès à grain grossier sont manifestement gravées. Si deux fragments qui se raccordent portent des gravures non déchiffrables, la troisième pièce est ornée d'un arrière-train fragmentaire. On y voit la 
partie postérieure de la ligne de dos, la naissance de la queue et le contour de la fesse parfaitement arrondie. La figuration d'un boviné est très vraisemblable (fig. 9.2).

Figure 9 - 1- Galet calcaire gravé, 2- Plaquette de grès ornée d'un arrière-train de boviné. Photos et croquis de lecture F. Plassard.

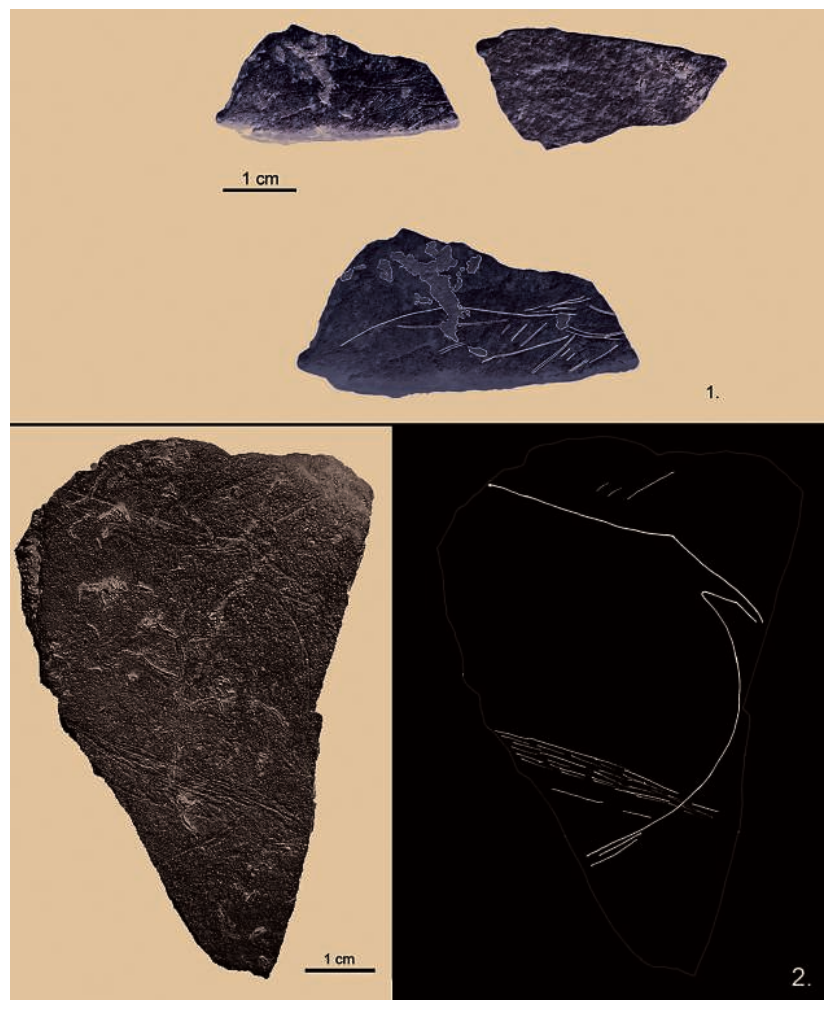

\section{3 - Les pièces inédites des ensembles remaniés}

\subsubsection{Le fragment de pièce festonnée (fig. 10)}


Figure 10 - 1- Fragment de pièce festonnée orné d'un salmonidé. 2- Observation sous loupe $(x 20)$. 3 et 4 - Observations sous loupe (x 20) de 2 saumons du lissoir dit de la "biche aux saumons ". Photos F. Plassard et L. Aurière.

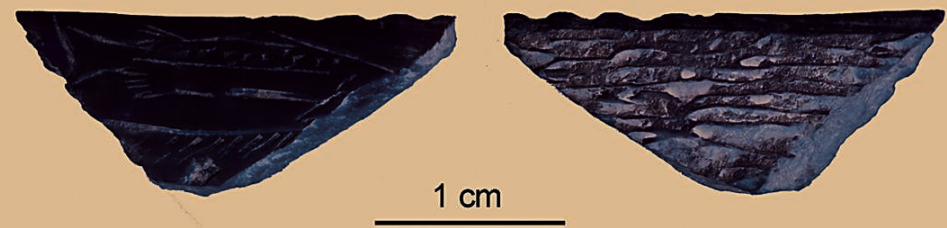

1.

\section{Matière : Os, Hémi-côte}

Dim : 2,26 x 9,1 x 0,26 cm

Section : Plane

Contour : Trois bords cassés

Profil : Rectiligne

Il a été réalisé sur hémi-côte. La face supérieure, constituée de tissu compact, a été régularisée par raclage et polissage, tandis que la face inférieure présente un tissu spongieux encore apparent. Un des bords est en partie orné de festons. Le décor est une représentation de salmonidé détaillée dont la tête est en partie manquante, par suite d'une cassure. On peut y observer les nageoires anale, adipeuse, dorsale et caudale, ainsi que la représentation de la ligne latérale. Des "ponctuations» en partie supérieure indiquent les tâches colorées typiques de l'espèce. Sous l'animal, les vestiges d'un autre élément graphique sont bien lisibles: une ligne droite agrémentée de courtes stries parallèles obliques.

Cette représentation de salmonidé n'est pas sans rappeler celles de la pièce dite de « la biche aux saumons » (fig. 18.3). Toutefois, les deux morceaux ne se raccordent pas. La morphologie du fragment et la présence de festons laissent entendre qu'il s'agit de deux pièces distinctes.

Les salmonidés de ces deux objets sont de facture graphique proche. Même si les saumons associés à la biche sont un peu plus géométrisés, on retrouve un schéma de construction formelle identique dans la forme en fuseau et certains détails anatomiques. Les petites dimensions de la surface contraignent l'auteur à des gestes saccadés, et la circulation de l'outil est beaucoup moins souple (fig. 10). 


\subsection{2 - Le complément du fragment d'hémi-côte aux ours (fig. 11)}

Figure 11 - 1- Complément du fragment d'hémi-côte aux ours avant restauration. 2 et 3- Avers et revers de la même pièce après restauration. Photos $F$. Plassard.

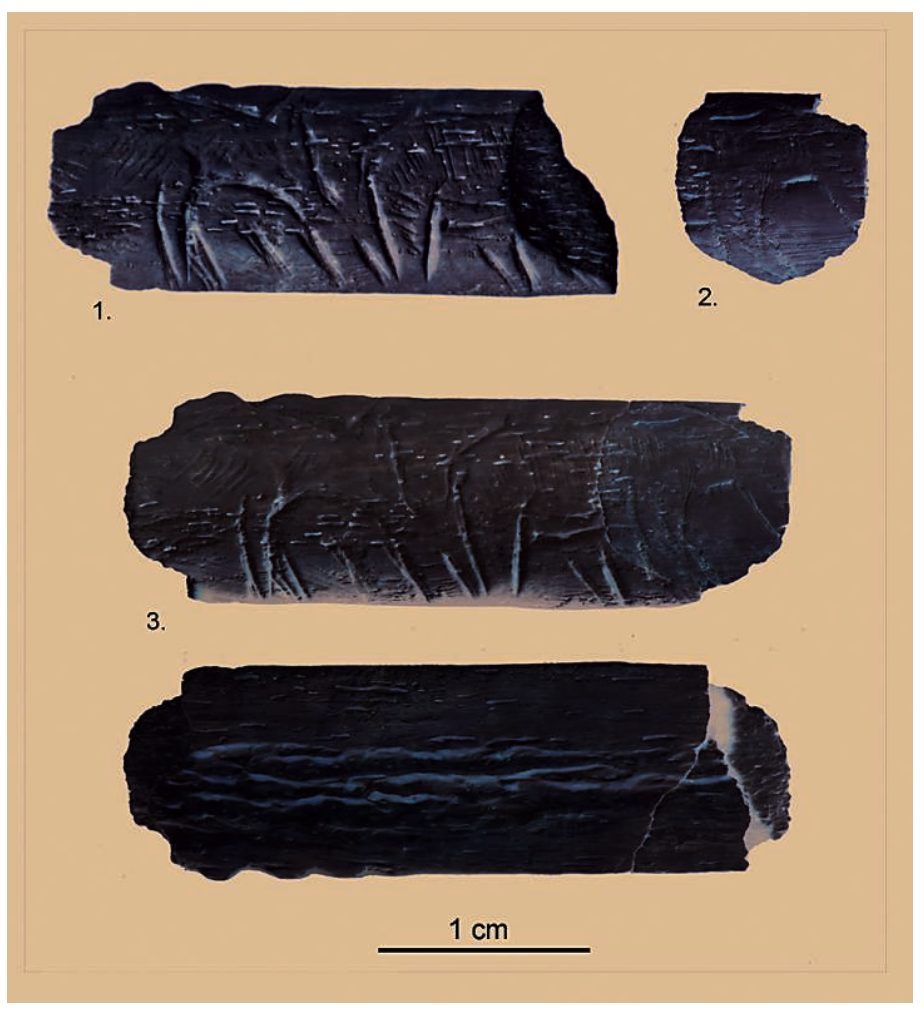

48 Matière : Os, Hémi-côte

$\operatorname{Dim}: 3,18 \times 1,02 \times 0,31 \mathrm{~cm}$

Section : Plano-convexe

Contour : Bords parallèles

Profil : Rectiligne

La découverte d'un nouveau fragment de seulement quelques millimètres (une nacelle) complète l'objet vers la droite et améliore la lecture d'un carnivore en profil droit pour lequel la partie antérieure manquait. Cette image avait été interprétée comme celle d'un «canidé ( ?)» par A. Roussot et C. Fritz (in Chauchat et al. 1999), mais c'est l'hypothèse d'une représentation d'ours évoquée par E. Man-Estier (2011) qui se trouve confortée par ce raccord. En effet, le sujet de droite est maintenant assez complet pour que soient identifiables, une queue courte ainsi qu'une ligne de garrot légèrement bossue et caractéristique des ursidés. Notons néanmoins que la tête du sujet de gauche semble bien peu épaisse pour celle d'un ours.

\subsection{3 - Le complément de la grande pendeloque au cervidé (fig. 12)}


Figure 12 - Complément de la grande pendeloque au cervidé. Le fragment inédit est indiqué par la flèche. Photo F. Plassard.

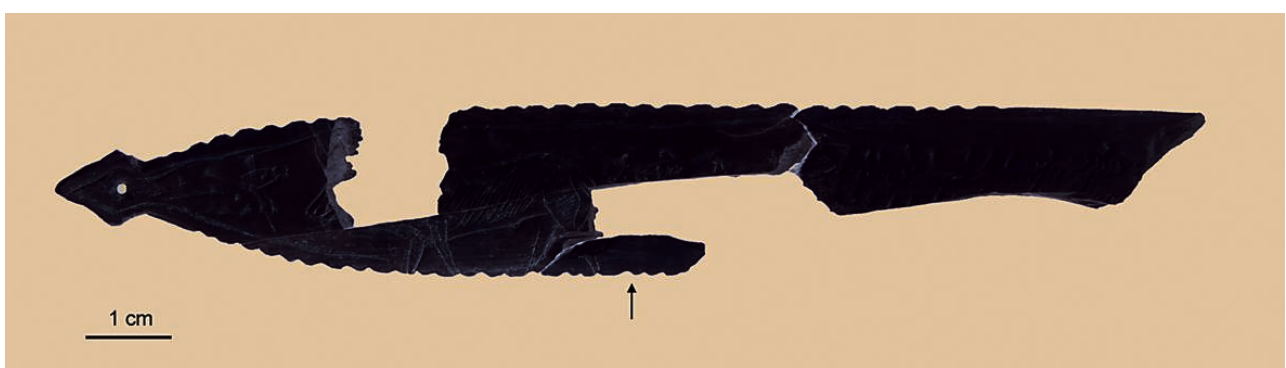

Matière : Os, Hémi-côte

Dim : $1,94 \times 0,49 \times 0,21 \mathrm{~cm}$

Section : Plano-convexe

Contour : Bords parallèles

Profil : Rectiligne

Un petit fragment dont le bord inférieur est festonné vient compléter la plus grande des pendeloques à bélière connues sur le site (Fritz et Roussot in Chauchat et al. 1999; Fritz 1999). Ce raccord, qui se positionne dans la partie inférieure de l'objet, complète la lecture de la patte arrière gauche du cervidé qui en constitue le décor principal.

\subsection{4 - Le complément de la pièce gravée de triangles (fig. 13)}

Figure 13 - Pendeloque gravée de motifs en triangles. Photos F. Plassard.
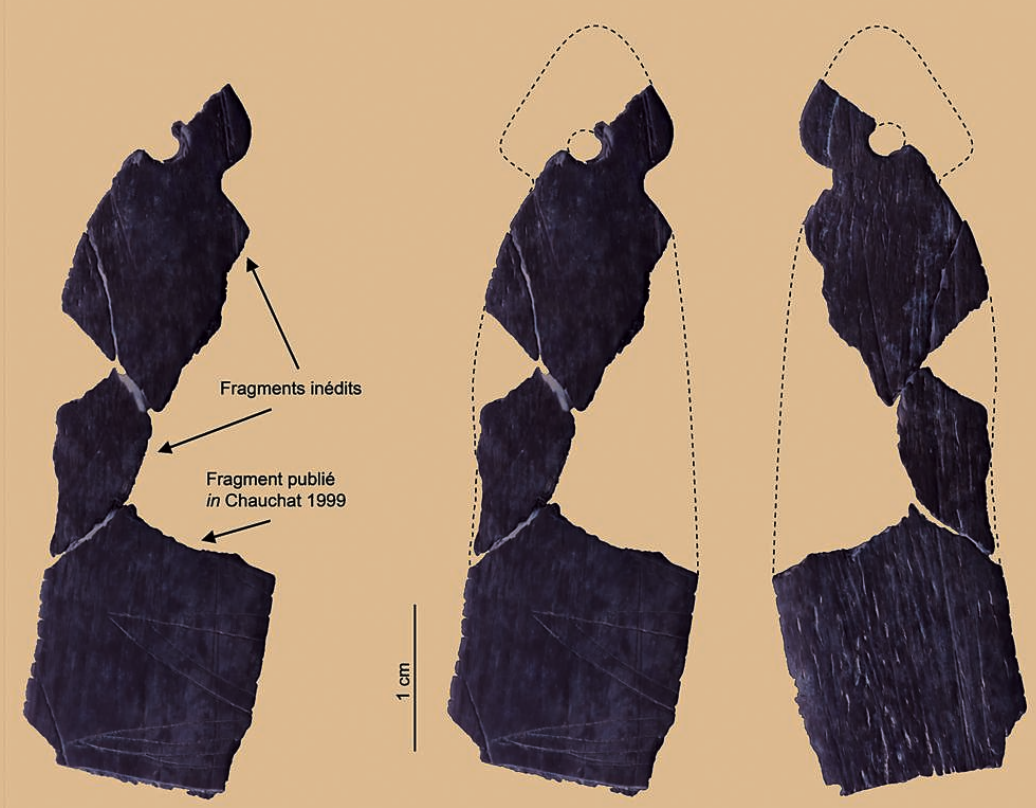

52 Matière : Os, Hémi-côte?

Dim : 4,1 x 1,4 x 0,1 cm

Section : Plano-convexe 
Contour : Bords parallèles

Profil : Rectiligne

53 A. Roussot et C. Fritz décrivent (in Chauchat et al. 1999) une petite plaquette d'os plat décorée de motifs en triangle et bordée de fines encoches. La fouille en 2002 d'une poche de sédiments remaniés par les fouilles clandestines a permis de découvrir trois fragments qui se raccordent à la pièce déjà connue. L'ensemble forme aujourd'hui une pendeloque à bélière fragmentaire dont la morphologie initiale est difficile à reconstituer mais dont nous proposons une esquisse. La partie proximale de la pendeloque ne semble pas ornée, exception faite de deux fines encoches sur le bord, similaires à celles décrites sur la partie distale.

\subsection{5 - Le fragment de pendeloque à bélière (fig.14)}

Figure 14 -1- Pendeloque à bélière. 2- Détail du décor autour de la bélière (x 40). 3- Détail de la grande pendeloque dont la bélière est similaire (x 40). Photos F. Plassard et $L$. Aurière.

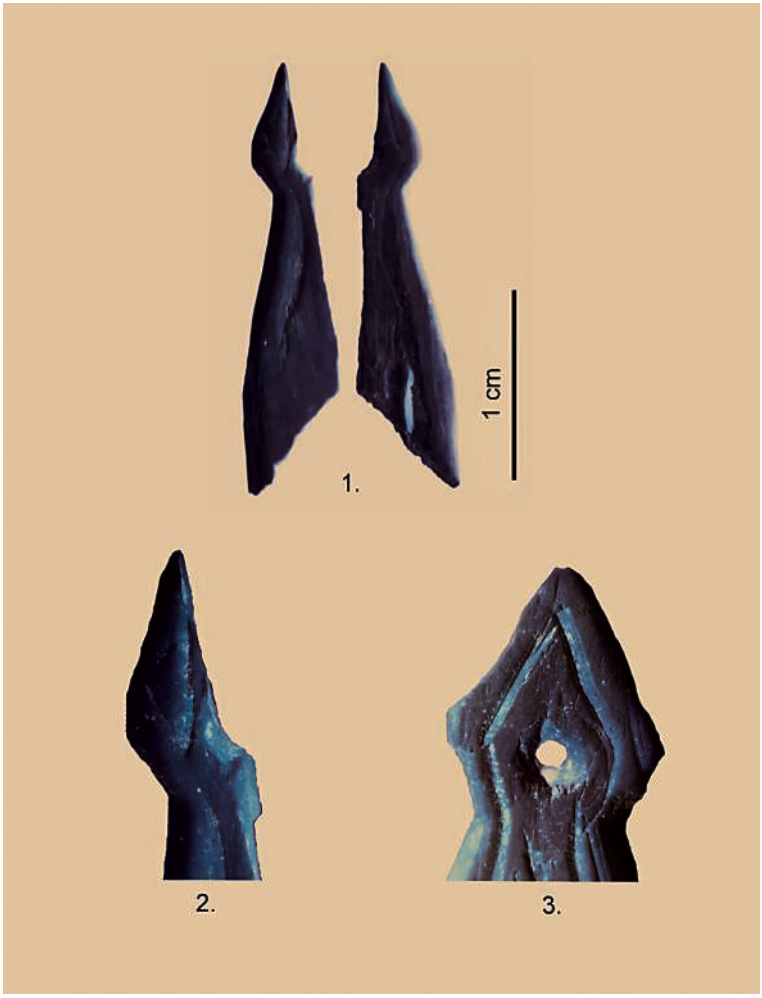

Matière : Os, Hémi-côte

$\operatorname{Dim}: 2,21 \times 0,5 \times 0,26 \mathrm{~cm}$

Section : Plane

Contour : Bords convergents en partie proximale

Profil : Rectiligne

Il s'agit d'un fragment d'hémi-côte travaillé avec une perforation encore identifiable. Les deux faces ont été mises en forme par raclage et polissage, tout comme l'échancrure du bord encore intact. Aucun décor n'est visible sur ce fragment, si ce n'est un trait qui souligne le contour de chacune des faces. Ce dernier rappelle un autre exemple de pendeloque à bélière (celle au cervidé, fig. 12), dont le contour est également souligné d'une gravure. 


\subsection{6 - La baguette à tubérosités (fig. 15.1)}

Figure 15 - 1- Baguette demi-ronde à tubérosités. 2- Baguette demi-ronde à décor en parenthèses. 3- Éclat de bois de cervidé orné de chevrons. 4- Fragment orné d'une pointe biconique. Photos $\mathrm{F}$. Plassard

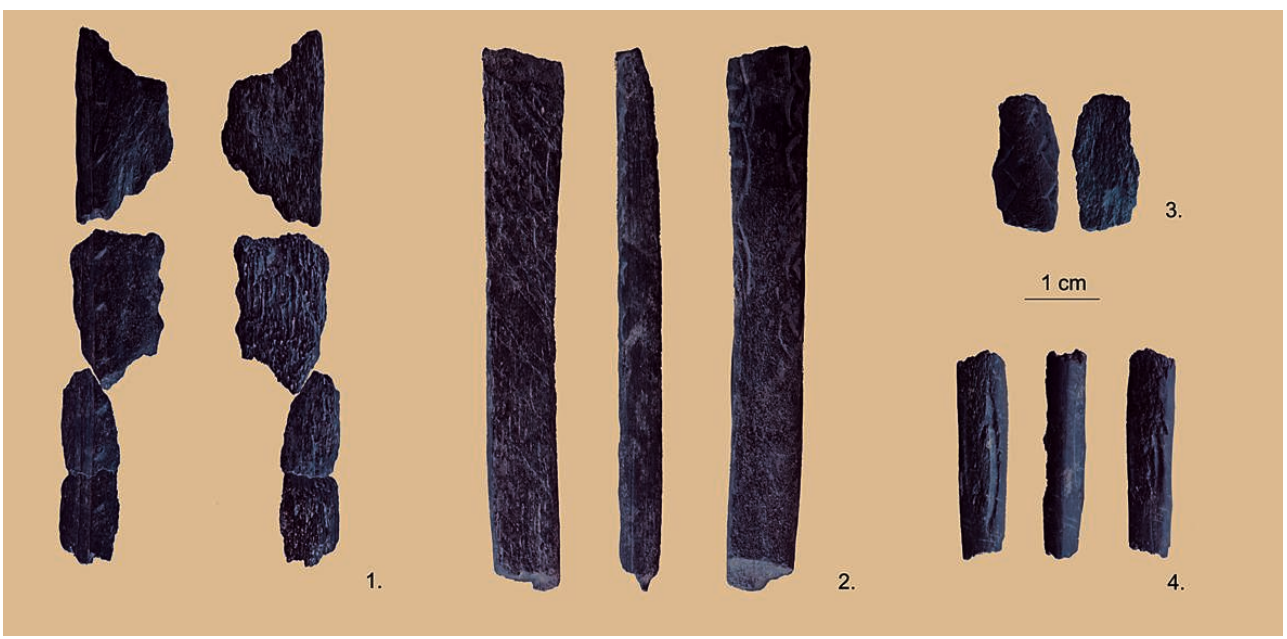

Matière : Bois de cervidé (très probablement bois de renne)

Dim : 4,7 × 1,2x 0,5 cm pour les trois fragments raccordés ; $2,7 \times 1,3 \times 0,4$ pour la partie isolée.

Section : Plano-convexe

Contour : Bords parallèles

Profil : Rectiligne

Cette pièce réalisée en bois de cervidé est constituée de quatre fragments. Trois d'entre eux ont été remontés. Le quatrième n'a pu faire l'objet d'un raccord physique mais il est fort probable qu'il appartient à cette baguette demi-ronde décorée de tubérosités. Le support a très probablement été débité par double rainurage. La face inférieure de l'objet est toujours pourvue de tissu spongieux et ne présente pas d'incisions d'encollage. Sur la face supérieure, il est possible de discerner au moins neuf tubérosités positionnées le long d'un méplat central. Les cassures qui affectent cet objet ne sont pas déterminables d'un point de vue fonctionnel.

\subsection{7 - Baguette demi-ronde décorée (ARA 98, salle du fond, fouille clandestine)}

(fig. 15.2)

Matière : Bois de cervidé (très probablement bois de Renne)

Dim : $7,4 \times 1,1 \times 0,4 \mathrm{~cm}$

Section : Plano-convexe

Contour : Bords parallèles

Profil : Rectiligne

L'état de conservation de cette baguette demi-ronde décorée en bois de cervidé est remarquable. L'objet est affecté d'une fracture en languette à l'une de ses extrémités et d'une cassure en dents de scie à l'autre. La pièce a été raclée sur la totalité de sa surface. Par la suite, la face supérieure de la baguette a été ornée, sur ses deux bords, de séries d'incisions profondes formant des motifs en «parenthèses ». Près du bord de la pièce, 
le centre de chaque parenthèse est orné d'un trait perpendiculaire à l'axe de la pièce ou d'un petit chevron. La face inférieure de la pièce comporte des incisions d'encollage qui sont nettement visibles.

\subsection{8 - Éclat de bois de cervidé (RH 18 A01 (1224)-465-ATR1) (fig. 15.3)}

Matière : Bois de cervidé

$\operatorname{Dim}: 1,8 \times 0,8 \times 0,3 \mathrm{~cm}$

Section : Biconvexe

Contour : Irrégulier

Profil : Rectiligne

Il s'agit d'un fragment de tissu compact de bois de cervidé. La nature même de l'objet dont provient cet éclat est inconnu. Sur la face supérieure, on distingue deux séries de trois chevrons alignés qui forment un décor en zigzag sur chacun des bords.

\subsection{9 - Base de pointe biconique (ARA 06, US 2003, remanié) (fig. 15.4)}

Matière : Bois de cervidé

$\operatorname{Dim}: 2,8 \times 0,6 \times 0,5 \mathrm{~cm}$

Section : Circulaire

Contour : Bords parallèles

Profil : Rectiligne

Réalisé sur bois de cervidé, cet objet de section circulaire est raclé sur la totalité de sa surface. Deux motifs en "parenthèses» ont été profondément gravés sur la face inférieure. Un décor semblable pourrait avoir été mis en place sur la face supérieure, qui est difficile à lire car altérée. Antérieurement à ces gravures marquées, de fines incisions, parallèles entre elles, ont été disposées transversalement à l'axe de la pièce. Elles évoquent des incisions d'encollage, laissant à penser que cet objet pourrait constituer la base d'une pointe (de projectile ?) biconique.

\subsubsection{0 - Les pièces sur support minéral}

Les ensembles sédimentaires remaniés n'ont livré que quatre pièces lithiques potentiellement ornées. Trois d'entre elles sont issues du tamisage des déblais de la fouille clandestine tandis que la dernière provient de l'US 2017 (ensemble remanié dont le matériel archéologique n'a aucune attribution chronologique sûre).

Aucune de ces pièces ne porte de décor déchiffrable. Il s'agit d'un galet de calcaire, dont la surface porte de nombreuses incisions parfois organisées en séries parallèles (fig. 16.1), de deux plaquettes de grès incisées de quelques traits énigmatiques et d'une plaquette de grès ornée de points diffus de couleur rouge. Si le galet de calcaire et la plaquette de l'US 2017 portent des gravures indiscutables (fig. 16.2), les autres pièces sont sujettes à caution : tracés peu nombreux et non organisés ou coloration diffuse peuvent être fortuits ou résulter de gestes uniquement techniques sans connotation symbolique. 
Figure 16 - 1- Galet de calcaire, gravé d'incisions fines et nombreuses, parfois organisées en séries parallèles (fouille clandestine). 2- Plaquette de grès gravée (US 2017). Photos F. Plassard.

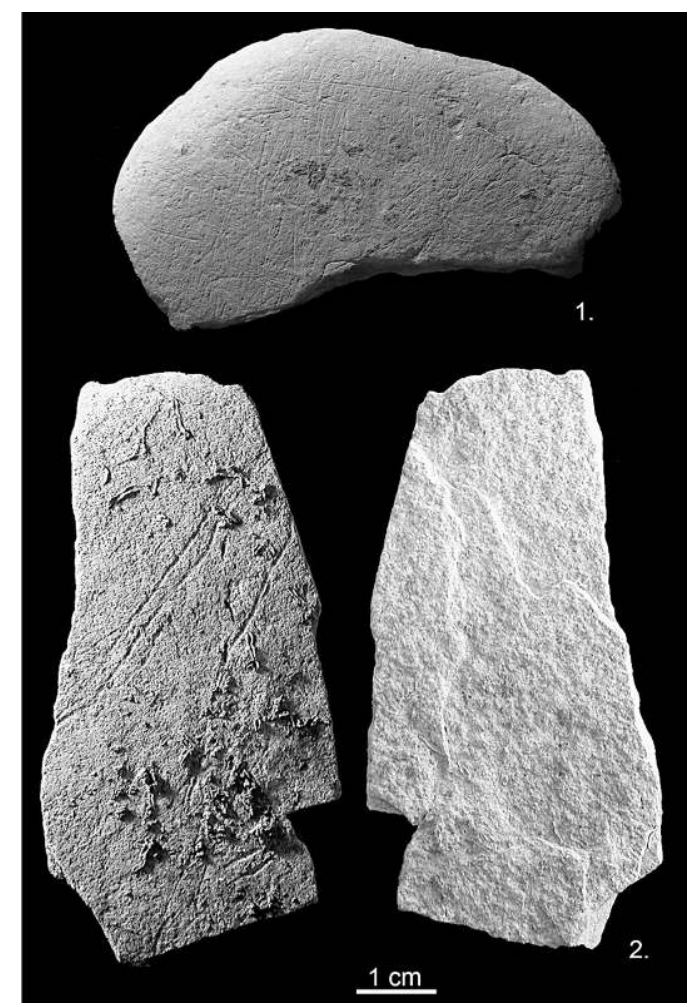

2.4 - Le raccord entre la fouille clandestine et un niveau en place (fig. 17) 
Figure 17 - 1- Lissoir festonné reconstitué à partir de 2 pièces de la fouille clandestine et d'une pièce découverte en stratigraphie. 2- Détail d'un des raccords (x 40). Photos F. Plassard et $L$. Aurière.

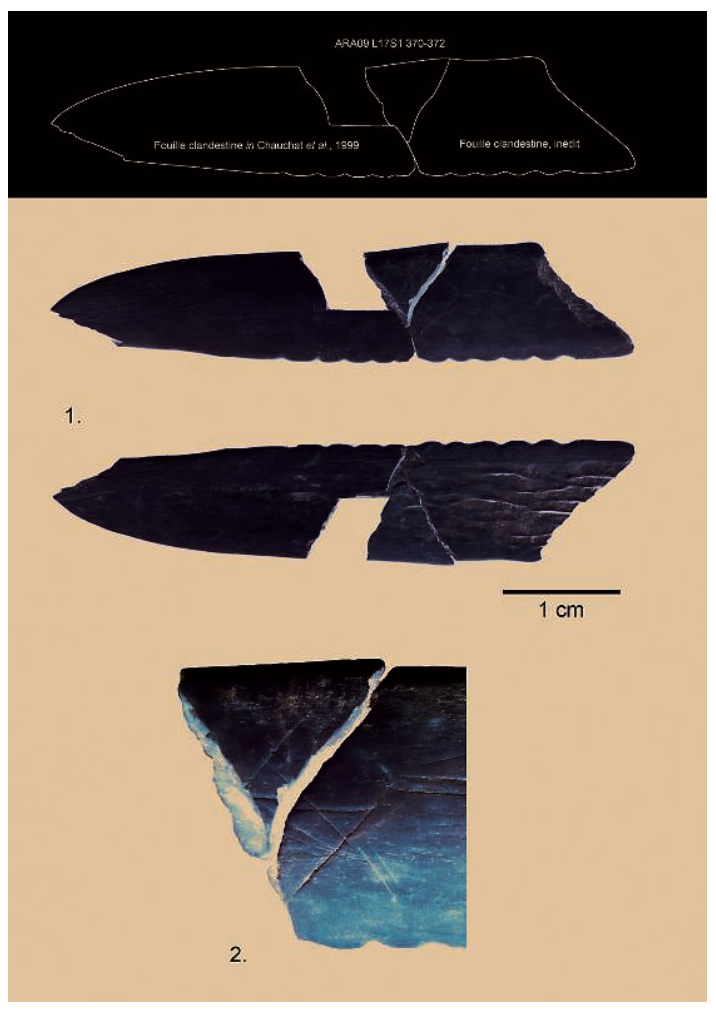

Il s'agit d'un raccord entre trois morceaux formant un lissoir à bord festonné réalisé sur hémi-côte. La partie distale est issue des fouilles clandestines et fut publiée en 1999 (Chauchat et al. 1999 : fig. 30). Elle mesure 3,14 cm de longueur, pour 0,95 cm de largeur et $0,14 \mathrm{~cm}$ d'épaisseur.

Le fragment proximal a été découvert dans les bacs de «restes de faune non déterminables » de la fouille clandestine, qui ont été réexaminés récemment. Il mesure $1,93 \mathrm{~cm}$ de long, 0,99 de large et 0,13 cm d'épaisseur. La face inférieure de l'hémi-côte est entièrement régularisée et la face supérieure présente de fines stries qui s'apparentent plus à du raclage qu'à de la gravure. Les festons se positionnent parfaitement dans le prolongement du premier fragment.

Le dernier fragment est de plus petites dimensions et se place en partie médiane, entre les deux précédemment décrits. Il remonte directement avec l'élément de droite, tandis qu'à sa gauche un morceau est toujours manquant. L'importance de ce petit fragment de $0,7 \times 0,7 \times 0,1 \mathrm{~cm}$ réside dans sa découverte en 2009 dans des niveaux d'occupation en place (US 2007E) de la salle du fond.

\section{3 - Analyses technologiques et stylistiques}

69 Le choix des matières osseuses employées est homogène. Mis à part les fragments de baguettes demi-rondes, l'os domine largement la série avec une utilisation prépondérante des côtes et des diaphyses d'os longs d'oiseaux.

70 Si toutes les œuvres d'art sur côte provenant de la fouille clandestine présentent des transformations importantes de la matière première (débitage afin d'obtenir une hémi- 
côte, façonnage poussé des faces inférieures et supérieures et réalisation de festons) dans le but d'obtenir des pendeloques ou des lissoirs, deux des pièces inédites (fig. 5 et 6) se singularisent par l'absence presque totale de préparation des supports. Seules quelques traces de raclage sur les bords laissent penser à une tentative de bipartition avortée.

71 La troisième pièce (fig. 7), réalisée probablement sur hémi-côte, porte des traces de préparation des surfaces par raclage en face supérieure. Elle semble se rapprocher d'avantage des spécimens de type pendeloque ou lissoir. Cependant, sa fracturation ne permet plus de la caractériser d'un point de vue typologique.

Les différences constatées dans la préparation des supports sont accentuées par celles que l'on détecte dans la technique de la gravure. Les figurations sur côte entière sont de dimensions importantes par rapport à la moyenne de celles des découvertes précédentes, connues pour faire partie des plus petites représentations de l'art mobilier magdalénien. En effet, ces dernières font entre 0,8 et $1,3 \mathrm{~cm}$. Elles sont détaillées et naturalistes, permettant une identification claire des espèces représentées (Cerf, Cheval, Saumon, Engoulevent,... (Fritz et Roussot in Chauchat et al. 1999 ; Fritz 1999). Au contraire, le Cheval et les canidés (fig. 5 et 6) mesurent entre 2,4 et $4,5 \mathrm{~cm}$ de long mais sont paradoxalement plus statiques et ne présentent aucun détail interne. Ces images présentent en outre des incohérences anatomiques dans les proportions, la mise en place de certains éléments ou l'absence de certains autres (œil, queue, etc.). Techniquement, des sorties de traits et des «broutages" liés à une mauvaise inclinaison de l'outil à la surface des supports sont décelables dans les tracés rectilignes et courbes.

73 La troisième pièce (fig. 7) se distingue des autres, tant par l'investissement technique préalable à la gravure que par sa précision graphique. Malgré son caractère fragmentaire, nous pouvons noter l'importance donnée aux détails avec la figuration du pelage, de l'œil et de l'oreille. Enfin, les tracés sont profonds et sans stigmates d'accident en dépit de leur longueur souvent millimétrique.

74 Le fragment de diaphyse gravée découvert dans le vestibule (ARA03 K24 2170, fig. 3) semble plutôt s'inscrire dans une chaîne opératoire de fabrication courte, sans préparation du support. Il est en revanche difficile de juger de la qualité technique et graphique de la gravure, tant l'objet est fragmentaire.

75 Les comparaisons entre les œuvres sur support organique et celles sur support minéral sont difficiles tant les secondes sont fragmentaires et peu explicites. Cependant, on peut remarquer la diversité des matériaux utilisés : grès, calcaire (identique ou non à l'encaissant de la grotte), galets et roche métamorphique. En outre, les supports ne présentent pas de traces de préparation. Les deux seules pièces offrant des images plus ou moins identifiables (une patte, gravée sur une pièce découverte dans le vestibule (fig. 4.2) et un arrière-train partiel (fig. 9.2) sur une plaquette provenant de la grotte) témoignent toutefois d'une bonne maîtrise technique. Dans les deux cas, les courbes gravées sont régulières, tandis que les différents segments du tracé se succèdent harmonieusement.

76 Si les nouvelles découvertes modifient quelque peu les caractéristiques technostylistiques des œuvres d'art de Bourrouilla, en revanche, le spectre des thèmes représentés demeure presque inchangé. En effet, le bestiaire des nouvelles découvertes (canidés, chevaux et cervidés) est largement en adéquation avec celui caractérisé dans la publication de 1999. Nous y retrouvons également l'association de deux animaux sur 
un même support, mais cette fois de la même espèce, contrairement à l'essentiel des découvertes précédentes. Ainsi, huit combinaisons animalières sont présentes : ours ?/ ours, cheval/oiseau, poisson/indéterminé, cervidé/poisson, oiseau/indéterminé, cerf/ cétacé, cheval/cheval, canidé/canidé. Au-delà de la variabilité importante des thèmes d'Arancou, un autre élément thématique se démarque: la présence d'espèces peu représentées habituellement dans l'art mobilier magdalénien, la sous représentation du cheval et du bison et la non-représentation du renne.

\section{4 - Les œuvres d'art dans leur contexte archéologique}

\section{1 - Attribution chrono-culturelle}

En l'état actuel des recherches, les déblais de la fouille clandestine ont livré 15 objets réalisés sur hémi-côtes, dont quatre pendeloques. La majorité des autres pièces font partie de la catégorie typologique des lissoirs (fig. 18). Leur largeur et leur épaisseur sont régulières d'une pièce à l'autre. Leur section est plano-convexe et leur profil rectiligne. Un façonnage poussé par raclage et polissage ne permet plus de lire les stigmates de débitage (Fritz et Roussot in Chauchat et al. 1999). Les fractures en languette en partie mésiale et la présence de stries multidirectionnelles associées à un poli en partie distale laissent supposer une utilisation en percussion posée sur des matières souples (Averbouh et Buisson 1996, 2003). La ressemblance dans la forme et la réalisation de ces pièces laisse envisager qu'elles constituent un lot issu d'une même production plus ou moins contemporaine.

Figure 18

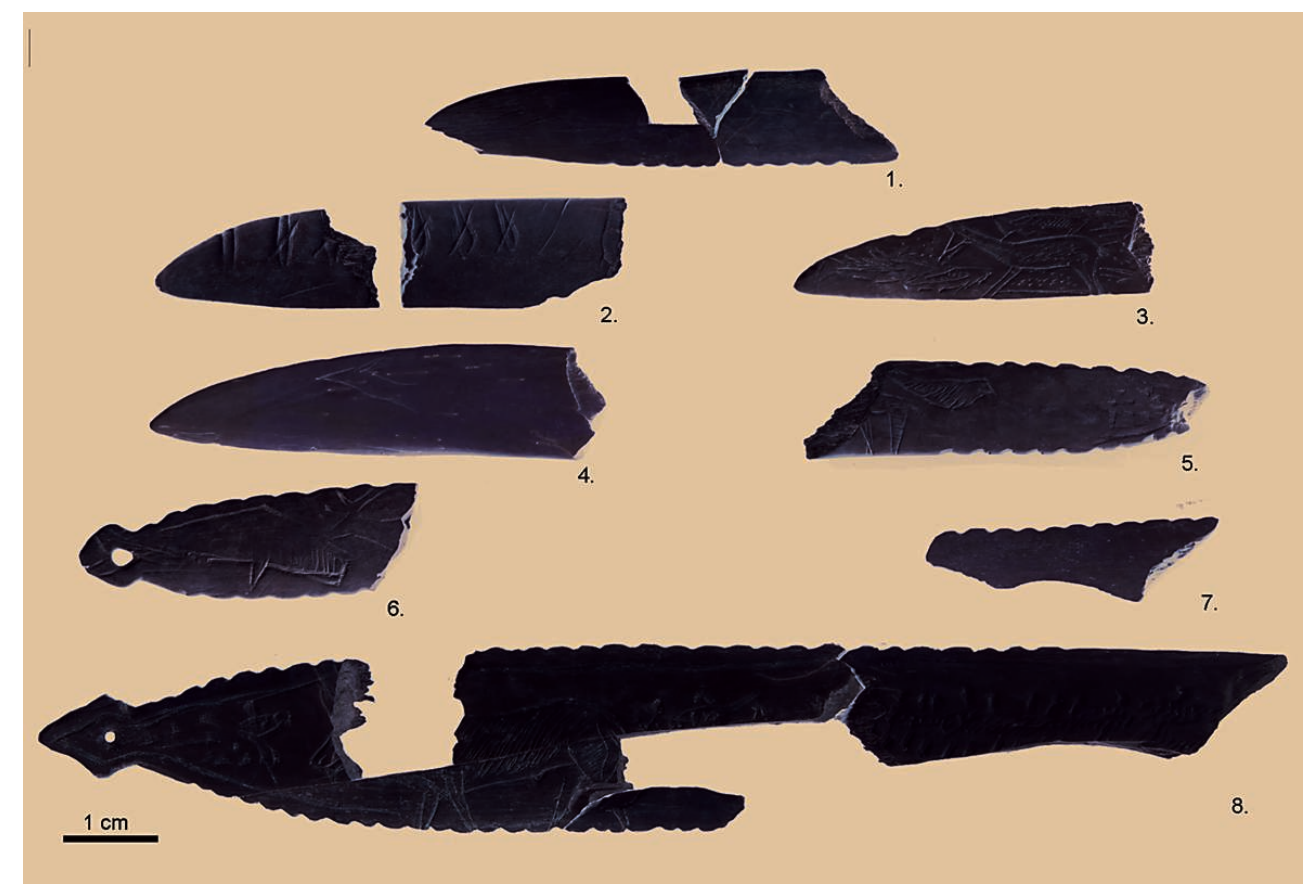

1- Lissoir festonné reconstitué à partir de deux pièces de la fouille clandestine et d'une pièce découverte en stratigraphie. 2- Lissoir orné de motifs fusiformes (anthropomorphes? ?). 3- Lissoir orné d'une biche et de saumons. 4- Lissoir orné d'un cervidé schématique. 5- Lissoir festonné et orné d'un engoulevent et d'un quadrupède indéterminé. 6- Pendeloque festonnée et ornée d'un cétacé et d'un cerf. 7- fragment de lissoir festonné. 8- Grande pendeloque festonnée et ornée d'un cervidé. Photos F. Plassard. 
Dans ce contexte, la découverte au sein d'un niveau bien conservé (l'US 2007E), à l'attribution chrono-culturelle claire, d'un petit fragment de lissoir participant à un raccord avec des pièces issues des déblais de la fouille clandestine est précieuse. En effet, elle conforte l'appartenance des objets de ce type au Magdalénien supérieur. De même, la découverte d'un tube gravé réalisé aux dépens d'une diaphyse d'os long de chouette Harfang au sein de l'US 2007AB s'accorde avec l'attribution au Magdalénien supérieur d'une autre diaphyse de Harfang ornée de deux chevaux et d'un oiseau. Plus largement, ce sont toutes les pièces d'art mobilier sur os découvertes à Arancou qui se voient ainsi attribuées plus sûrement au Magdalénien supérieur.

Pour l'extérieur et le vestibule, les données sont plus rares mais l'appartenance de tous les vestiges ornés au Magdalénien supérieur est assurée.

Néanmoins, à ce jour, aucune pièce ornée sur bois de cervidé n'a été découverte dans les niveaux du Magdalénien supérieur. Les fragments de baguettes demi-rondes issus des déblais de la fouille clandestine, et sans doute rapportables au Magdalénien moyen (Feruglio 1992), trouveront probablement leurs homologues dans des niveaux sousjacents à l'US 2007, et/ou à l'ensemble C.

\section{2 - Distribution spatiale des activités et structuration de l'espace}

81 Cependant, la présence au sein de l'US 2007E du fragment de lissoir, de la côte gravée de deux canidés et de plaquettes de grès ornées de tracés indéchiffrables conduit à considérer que la série des œuvres d'art de ce niveau n'est pas totalement homogène, tant du point de vue des supports que de celui de la chaîne opératoire de production (façonnage, gravure). De même, la coexistence dans l'US 2007AB du tube en os d'oiseau, de la côte aux chevaux, de la plaquette en grès gravée de l'arrière-train d'un boviné et de plaquettes de calcaire identique à l'encaissant, ornées de fines gravures inintelligibles, reflètent encore la pluralité de la production graphique attachée à ce niveau d'occupation. Au sens large, l'US 2007, attribuable de façon certaine au Magdalénien supérieur, fournit un spectre varié d'œuvres d'art où voisinent les supports lithiques et organiques, les supports bruts et préparés, les représentations soignées et maîtrisées et d'autres plus simples et malhabiles.

L'identification de vestiges caractéristiques du travail de l'os et des bois de cervidés (supports bruts, ébauches, déchets) permet d'argumenter clairement en faveur de la production sur place d'une partie au moins de l'armement (têtes de projectile) et de l'outillage (aiguilles à chas) dans ces matériaux (Chauvière, étude en cours). En est-il de même de l'art mobilier sur supports organiques? La proportion relativement forte, dans le site d'Arancou, de petits lissoirs décorés et de pendeloques à bélière, par rapport à ce que l'on observe dans d'autres gisements de la chaîne pyrénéo-cantabrique (voir ci-dessous) pourrait tout autant constituer un argument en faveur d'une fabrication sur place que témoigner d'une introduction d'objets à l'état fini. Trancher entre ces deux options est pour l'instant délicat dans la mesure où il n'est pas aisé d'établir une distinction nette et systématique entre des chaînes opératoires spécifiquement dédiées à la confection d'objets décorés, et des chaînes opératoires de fabrication de l'équipement matériel au sein desquelles l'art mobilier ne serait qu'un produit accessoire (la différence résidant uniquement dans la mise en place ou non d'une ornementation). L'ubiquité de certains déchets de fabrication produits ajoute encore à la difficulté actuelle. La question se pose de manière cruciale pour les petits 
lissoirs décorés réalisés sur hémi-côte et les éléments potentiellement inféodés à la sphère des projectiles telles que les baguettes demi-rondes (fig. 15.1 et 15.2) ou la pointe biconique (fig. 15.4). Elle se pose également pour les os d'oiseaux. Doit-on par exemple associer d'emblée l'ulna de chouette Harfang décoré (fig. 8) décrit dans cet article à l'exploitation intensive de l'avifaune (Eastham 1998; Eastham in Chauchat et al. 1999) ? L'abondance des restes osseux aviaires dans la grotte et des traces qu'ils portent autorise une association contextuelle et théorique, sans que la possibilité d'une pièce introduite à l'état fini dans le gisement ne soit exclue.

Sur l'ensemble des pièces ornées découvertes depuis 1998, onze fragments ornés ont été découverts en zone extérieure ou dans le vestibule. Seulement quatre présentent des éléments de décor déchiffrables : trois sur matière lithique et un sur fragment de diaphyse. Ces derniers sont cependant associés à un secteur reconnu à la fouille comme une zone liée à un foyer, déjà décrite comme ayant accueilli des activités d'entretien des armes de chasse (Dachary et al. 2008).

La salle du fond, quant à elle, rassemble 40 pièces ornées. Trente et une ont été réalisées sur matières osseuses, auxquelles doivent être ajoutés les restes fauniques portant des traces anthropiques isolées, très nombreux et dont la moyenne des longueurs ne dépasse pas $2 \mathrm{~cm}$. Malgré leur petite taille, ces fragments, notamment ceux sur os d'oiseaux, portent des incisions appuyées, qui pourraient être d'ordre davantage " artistique » que strictement technique.

Si la plaquette de grès gravée d'un arrière-train de boviné est clairement associée à un foyer identifié dans l'US $2007 \mathrm{AB}$, le tube en os d'oiseau et la côte aux chevaux ont été découverts dans ce qui semble être une zone de rejet. La côte aux canidés présente pour sa part des traces de chauffe qui laissent imaginer, si cette chauffe n'est pas fortuite, qu'elle a pu être recyclée comme combustible. Il est alors possible de s'interroger sur le statut de ces objets ornés, à la fonction énigmatique et qui sont abandonnés et/ou détruits une fois achevés. La démarche de destruction volontaire ou de réutilisation de l'objet orné est couramment observée au Magdalénien (de Beaune 1989, 1996, 1997 ; Tosello 2003, 2005). Le décor ne semble alors n'avoir eu qu'une valeur d'instant, peutêtre juste au moment de sa réalisation (Fritz et Pinçon 1989).

\section{Conclusions}

La découverte de nouvelles pièces ornées, en contexte stratigraphique pour certaines d'entre elles, permet de mieux appréhender la production artistique de Bourrouilla qui s'avère plus complexe que ne le laissaient penser les pièces décrites dans la publication de Chauchat et al. 1999. Trois résultats majeurs sont identifiables.

Le premier d'entre eux concerne les matériaux et leur mise en œuvre. Ils sont en effet plus variés que ce que laissaient percevoir les études antérieures, intégrant des supports lithiques et des pièces osseuses brutes. Cependant, en dépit d'une variabilité en termes de matières premières, de technique et de thèmes, l'étude techno-stylistique de la production artistique démontre qu'une partie de la production est homogène. Ce constat rejoint les conclusions de la première étude consacrée à ces vestiges (Fritz et Roussot in Chauchat et al. 1999). Les similitudes typologiques des lissoirs et des pendeloques à bélière à bords festonnés, tout comme le style des décors (petites 
dimensions, même technique de remplissage pour figurer le pelage et présence de motifs « punctiformes ») (fig.18), sont incontestables.

La série totalise aujourd'hui 17 pièces travaillées et/ou décorées réalisées sur côtes. Cet échantillon, auquel doivent être ajoutées des pièces en cours de transformation, est suffisant pour documenter au moins la phase de façonnage des matériaux puis de mise en place du décor et/ou de l'utilisation.

Les objets ornés réalisés sur côte montrent deux gestions distinctes du même matériau. D'une part, nous observons une production dont l'ensemble des opérations de mise en forme mène à la création d'objets élaborés. D'autre part, nous constatons la présence d'objets à faible transformation technique : la mise en place du décor est la principale modification et leur fonction est inconnue (Aurière 2009, sous presse).

La première de ces productions est associée à une gravure de qualité en termes techniques et graphiques, alors que la seconde est caractérisée par des figurations plus sommaires.

91 Cette distinction laisse supposer que ces objets n'ont pas été produits par les mêmes personnes, voire qu'ils avaient des fonctions différentes. Si la fonction des outils et celles de l'ornementation des lissoirs et des pendeloques peuvent être facilement envisagées, celle(s) des objets à faible transformation technique reste(nt) plus énigmatique(s). La présence assez récurrente sur ces pièces de dessins aux tracés maladroits pourrait laisser envisager qu'il s'agit de supports dévolus à l'apprentissage de la gravure (Fritz 1999 ; Rivero 2010 ; Aurière 2012).

Dans un autre ordre d'idées, la découverte de nouvelles pièces ornées en contexte stratigraphique permet leur attribution au Magdalénien supérieur. Elle conduit à attribuer à la même phase chronoculturelle des pièces comparables mises au jour dans les déblais de la fouille clandestine.

Enfin, les dernières découvertes permettent d'appréhender la place des productions symboliques au sein de l'habitat. Au vu des actuelles données de fouille concernant la répartition des objets dans le gisement, il semble que la salle du fond soit un secteur privilégié pour la production d'œuvres d'art. Y coexistent, dans les mêmes niveaux d'occupation, des pièces ornées sur supports lithiques et osseux, préparés ou non. Néanmoins, pour le moment, une aire d'activité spécialisée pour la création artistique n'est pas clairement identifiable, les pièces ayant plutôt été découvertes abandonnées à proximité du foyer ou en zone de rejet. L'abandon, la destruction et la réutilisation clairement volontaire de ces objets nous laissent entrevoir qu'ils devaient avoir un statut, une valeur, bien plus complexe qu'il n'y paraît.

Le tamisage des déblais de la fouille clandestine au début des années 1990 a été l'occasion de découvrir un important lot de pièces sur hémi-côtes. Ces vestiges, souvent ornés de miniatures, trouvent leur équivalent dans quelques gisements pyrénéocantabriques (El Pendo, Isturitz, Espalungue Lortet, le Mas d'Azil, La Vache, ...) (Chollot 1964 ; Thiault et Roy 1996; Baffier et al. 2003, etc.). Si, à La Vache et Isturitz, l'attribution au Magdalénien supérieur de ces objets ne pose pas de problème, les pièces mises au jour dans les autres gisements, venant de fouilles anciennes, ne peuvent être que rapportées au Magdalénien.

95 Le raccord décrit ci-dessus qui associe deux pièces issues de la fouille clandestine avec un élément mis au jour dans les niveaux en place, donne un contexte archéologique clair aux hémi-côtes façonnées de Bourrouilla et permet par conséquent de proposer 
une attribution chronologique pour les pièces homologues découvertes dans d'autres sites.

Fort de ce cadrage chronologique, il devient dès lors pertinent d'aborder des questions de territoire, de mobilité et de contact entre les groupes humains, notamment par l'étude des analogies formelles et thématiques des objets d'art (Conkey 1987; Sauvet et al. 2008 ; Fritz et al. 2007 ; Rivero 2010). Si la production d'objets sur côtes de type petits lissoirs ou "pendeloque" se retrouve, nous l'avons vu, tout le long de la chaîne pyrénéo-cantabrique, leur nombre est toujours réduit. L'effectif relativement élevé de ces objets à Arancou nous semble donc disproportionné au vu de la petite taille du gisement. Cela pose la question de la place de ce site dans le contexte plus général du Magdalénien des Pyrénées occidentales (Dachary 2002, 2009 ; Dachary et al. 2008). En d'autres termes, cette abondance relative de lissoirs/pendeloques est-elle le reflet d'une activité pratiquée plus particulièrement à Arancou ou s'agit-il d'une production ubiquiste mieux documentée à Arancou que dans d'autres gisements contemporains? Est-ce un comportement technique propre à une phase très brève du Magdalénien supérieur, et aujourd'hui préservé dans un petit nombre de sites?

Seule une reprise du matériel issu de fouilles anciennes d'autres gisements et une étude techno-stylistique et fonctionnelle des productions sur côtes permettra de mieux percevoir le degré d'homogénéité de ces objets comparables d'un point de vue morphologique.

\section{BIBLIOGRAPHIE}

AURIÈRE L. 2009 - Approche technologique de l'art mobilier paléolithique en matières osseuses : premières recherches sur la phase de préparation. In : L'Art des Sociétés Préhistoriques, Rencontres Internationales Doctorants et Post-doctorants 1ère édition, Toulouse avril 2008, Préhistoire, Art et Sociétés, LXIV, p. 7-15.

AURIÈRE L. 2012 - L'art mobilier magdalénien, du support au décor. Les choix technologiques et leurs implications dans l'élaboration des objets ornés en matières osseuses. Etude de cas dans la vallée de l'Aveyron : les gisements de Plantade, Lafaye, Montastruc et Courbet. Thèse de Doctorat, Université Toulouse le Mirail, 2 tomes.

AURIÈRE L. sous presse - Réflexions autour des choix technologiques dans l'art mobilier magdalénien en matières osseuses. In : E. López-Montalvo et M. Sebastián López (coord.) El Legado Artístico de las sociedades prehistóricas.

AVERBOUH A., BUISSON D. 1996 - Approche morpho-fonctionnelle des objets nommés "lissoirs" : essai d'établissement d'une fiche analytique théorique. Antiquités Nationales, 28, p. 41-46.

AVERBOUH A., BUISSON D. 2003 - Les lissoirs. In : J. Clottes et H. Delporte (Dir.), La grotte de la Vache (Ariège). Fouilles Romain Robert. I- Les occupations du Magdalénien, Paris, Coédition CTHS-RMN, vol. 1, p. 309-324. 
BAFFIER D., BUISSON D., DELPORTE H., FRITZ C., GUY E., KANDEL D., MONS L., SIMONNET R., TOSELLO G., WELTÉ A.-C. 2003 - Lissoirs. In : J. Clottes et H. Delporte (Dir.), La grotte de la Vache (Ariège). Fouilles Romain Robert. II- L'art mobilier, Paris, Coédition CTHS-RMN, vol. 2, p. 279-319.

BEAUNE S. A. (de) 1989 - Fonction et décor de certains ustensiles paléolithiques en pierre. L'Anthropologie, 93(2), p. 574-584.

BEAUNE S. A. (de) 1996 - L'art au Paléolithique supérieur : éphémère ou durable ?. Antiquités Nationales, 28, p. 135-138.

BEAUNE S. A. (de) 1997 - Les galets utilisés au Paléolithique supérieur. Paris, CNRS éditions (Suppléments à Gallia Préhistoire, 22), 298 p.

CHAUCHAT Cl. (dir.), FONTUGNE M., HATTE C., DACHARY M., BONNISSENT D., CHAUVIÈRE F.-X., ROUSSOT A., FRITZ C., FOSSE Ph., EASTHAM A., MARTIN H., LE GALL O., GAMBIER D. 1999 L'habitat Magdalénien de la grotte Bourrouilla à Arancou (Pyrénées Atlantiques). Gallia Préhistoire, 41, p. 1-151.

CHOLLOT M. 1964 - Musée des Antiquités Nationales : collection Piette. Art mobilier préhistorique. Paris, Editions des Musées Nationaux, 479 p.

CONKEY M. W. 1987 - L'art mobilier et l'établissement de géographies sociales. In : J. Clottes (Dir.), L'art des objets au Paléolithique : les voies de la recherche, Colloque de Foix-Le Mas d'Azil, Paris, Ministère de la Culture, p. 163-17.

DACHARY M. 2002 - Le Magdalénien des Pyrénées occidentales, Thèse de Doctorat nouveau régime, Université de Paris $\mathrm{X}, 2$ tomes.

DACHARY M. 2005 - La grotte de Bourrouilla à Arancou (Pyrénées-Atlantiques) : bilan des fouilles 2002 à 2004. Archéologie des Pyrénées occidentales et des Landes, 24, p. 7-18.

DACHARY M. 2009 - Les Magdaléniens des Pyrénées occidentales. Réflexions sur l'exploitation d'un territoire. In : Djindjian F. et Oosterbeek L. (Eds). Symbolic Spaces in Prehistoric Art, Territories, travels and site locations. Proceedings of the XV. Congress of the U.I.S.P.P., Session C28, Archaeopress, 5 fig., p. 39-45.

DACHARY M. (dir.), CHAUVIÈRE F.-X., COSTAMAGNO S., DAULNY L., GAMBIER D., LAROULANDIE V. 2006 - « Les Magdaléniens à Duruthy », catalogue d'exposition (7 octobre-10 décembre 2006), Hastingues, Centre Départemental du Patrimoine, $188 \mathrm{p}$.

DACHARY M., CHAUVIÈRE F.-X., COSTAMAGNO S., DAULNY L., EASTHAM A., FERRIER C., FRITZ C. 2008 - La grotte Bourrouilla à Arancou : une puissante stratigraphie au service de la perception de la fin du Magdalénien pyrénéo-cantabrique. In : J. Jaubert, J.-G. Bordes, I. Ortega (dir.), Les sociétés paléolithiques dans un Grand Sud-Ouest : nouveaux gisements, nouvelles méthodes, nouveaux résultats. Journée SPF, Bordeaux, 24-25 novembre 2006, Paris, Société Préhistorique Française (mémoire 47), p. 355-370.

DACHARY M., PLASSARD F., MERLET J.-Cl., BONNET-JACQUEMENT P., CHAUVIÈRE F.-X. sous presse - L'Azilien des Pyrénées occidentales : Vers une révision de l'attribution chrono-culturelle des séries archéologiques. In : C. Cretin, O. Ferullo, J.-C. Castel (org.) « Deuxième moitié et fin du Paléolithique supérieur. Pour une confrontation entre le modèle classique et les perceptions interdisciplinaires actuelles sur le thème des unités, continuités et discontinuités ». Acte de la session F du XXVIIe Congrès Préhistorique de France. Paris, Société préhistorique française.

DACHARY M., MERLET J.-Cl., MIQUÉOU M., MALLYE J.-B., LE GALL O., EASTHAM A. 2013 - Les occupations mésolithiques de Bourrouilla à Arancou (Pyrénées-Atlantiques). Paleo, ce volume. 
EASTHAM A. 1998 - Magdalenians and snowy owls : bones recovered at the Grotte de Bourrouilla, Arancou (Pyrénées-Atlantiques). Paleo, 10, p. 95-107.

FERUGLIO V. 1992 - Fiche baguettes demi-rondes. In : H. Camps-Fabrer (Dir.), Fiches typologiques de l'industrie osseuse préhistorique. Cahier V. Bâton percé, baguettes. Treignes, CEDARC, p. 71-83.

FRITZ C. 1999 - La gravure dans l'art mobilier magdalénien; du geste à la représentation. Paris, Editions de la Maison des sciences de l'Homme (Documents d'Archéologie Française, 75), 216 p.

FRITZ C., PINÇON G. 1989 - L'art mobilier paléolithique : valeur d'instants, de la création à la destruction. In : J-P. Mohen (Dir.), Le temps de la Préhistoire, Dijon, Archéologia-Société préhistorique française, t. 2, p. 161-163.

FRITZ C., TOSELLO G., SAUVET G. 2007 - Groupes ethniques, territoires, échanges : la « notion de frontière » dans l'art magdalénien. In : N. Cazals, J. González Urquijo et X. Terradas (dir.), Frontières naturelles et frontières culturelles dans les Pyrénées préhistoriques, Université de Cantabria, Santander, p. 165-181.

LEROI-GOURHAN A. 1943 - Evolution et techniques I ; L'homme et la matière. Paris : Editions Albin Michel, 348 p.

MAN-ESTIER E. 2011 - Les Ursidés au naturel et au figuré pendant la Préhistoire, Liège, Université de Liège (ERAUL, 127), 801 p.

PELEGRIN J., KARLIN Cl., BODU P. 1988 - « Chaînes opératoires » : un outil pour le préhistorien, In : J. Tixier (dir.), Technologie préhistorique, Paris, Éditions du CNRS (Notes et monographies techniques, 25), p. 55-62.

PERLES C. 1991 - Économie des matières premières et économie du débitage : deux conceptions opposées ? In : 25 ans d'études technologiques en Préhistoire, bilan et perspectives, Juan-les-Pins, APDCA, p. 35-45.

PÉTILLON J.-M. 2007 - Les pointes à base fourchue de la zone pyrénéo-cantabrique : un objet à la charnière entre Magdalénien moyen et Magdalénien supérieur ? In : Frontières naturelles et frontières culturelles dans les Pyrénées préhistoriques, N. Cazals, J. González Urquijo, X. Terradas (dir.), Santander, Ediciones de la Universidad de Cantabria, p. 245-264.

RIVERO O. 2010 - La movilidad de los grupos humanos en el Magdaleniense de la Región Cantábrica y los Pirineos : Una visión a través del arte. Thèse de Doctorat, Université de Salamanque, 2 tomes.

SAUVET G., FORTEA J., FRITZ C., TOSELLO G. 2008 - Crónica de los intercambios entre los grupos humanos paleolíticos. La contribución del arte para el periodo 20.000-12.000 años BP., Zephyrus, LXI (1), p. 33-59.

SERANGELI J. 2003 - La zone côtière et son rôle dans les comportements alimentaires des chasseurs-cueilleurs du Paléolithique supérieur. In : Patou-Mathis, M., Bocherens, H. (Eds.), Le Rôle de l'Environnement dans les Comportements des Chasseurs-cueilleurs Préhistoriques. BAR International Series 1105, Oxford, p. 67-82.

STUIVER M., REIMER P.J. 1993 - Extended 14C data base and revised CALIB 3.0 14C Age calibration program. Radiocarbon, 35(1), p. 215-230.

SZMIDT C., LAROULANDIE V., DACHARY M., LANGLAIS M., COSTAMAGNO S. 2009 - Harfang, Renne et Cerf : nouvelles dates $14 \mathrm{C}$ par SMA du Magdalénien supérieur du Bassin aquitain au Morin (Gironde) et Bourrouilla (Pyrénées-Atlantiques). Bulletin de la Société préhistorique française, 106(3), p. 583-601. 
THIAULT M.-H., ROY J.-B. 1996 - L’art préhistorique des Pyrénées. Paris : Réunion des Musée Nationaux, $371 \mathrm{p}$.

TOSELLO G. 2003 - Pierres gravées du Périgord Magdalénien. Art, symboles, territoires. Paris, CNRS éditions (Suppléments à Gallia Préhistoire, 36), 590 p.

TOSELLO G. 2005 - Un contexto social para el arte mueble paleolitico en Franciado (Eds.), La materia del lenguaje prehistórico : el arte mueble paleolítico de Cantabria en su contexto, Santander, Gobierno de Cantabria, p. 53-65.

\section{RÉSUMÉS}

Depuis le début de la fouille programmée en 1998, les découvertes d'art mobilier dans le gisement Magdalénien de Bourrouilla (Arancou, Pyrénées-Atlantiques) se sont multipliées. Ainsi, ce sont dix-neuf pièces inédites et trois raccords avec des pièces déjà connues qui sont présentés d'un point de vue thématique, stylistique et technique. La découverte d'œuvres d'art sur support lithique, ainsi que celle de gravures sur des pièces osseuses n'ayant subi que peu de préparations préalables à la gravure, éclairent d'un jour nouveau la production symbolique de ce site jusque-là caractérisée par la présence d'une série de lissoirs très standardisés, réalisés sur des hémi-côtes intensément façonnées.

La découverte de neuf de ces objets au sein des occupations magdaléniennes, en contexte stratigraphique, permet de proposer une attribution chrono-culturelle des pièces inédites et de certaines de celles issues de la fouille clandestine. Par ailleurs, les données contextuelles et technologiques obtenues conduisent à engager une réflexion sur les modalités de production de ces objets, sur leur place au sein du gisement et leur fonction au sein du ou des groupes humains qui les ont réalisés et utilisés.

Since the beginning of the excavation programme in 1998 of the Magdalenian levels of Bourrouilla (Arancou, Pyrénées-Atlantiques), the discoveries of mobile art have multiplied. There are nineteen new pieces and three fragments completing artefacts known from the 90's presented from a thematic, stylistic and technical approach. The discovery of decorated works on lithic supports and the discovery of engravings on bones without preliminary preparation for the engraving give a new dimension to the symbolic production of this site. In fact, until now, the portable art has been characterized by the presence of a series of very standardized, polishingtools on much-worked half ribs.

The discovery of nine of these objects in the stratified Magdalenian levels allows us to propose a chronocultural attribution for the new pieces as well as for some of those from the clandestine excavations. The contextual data obtained during the excavation and the technological data offer new perspectives on the understanding the modalities of the production of these objects, on their place in the site and on their function for the human groups.

\section{INDEX}

Mots-clés : Magdalénien supérieur, art mobilier, contexte stratigraphique, industrie osseuse, technologie osseuse, Pyrénées occidentales

Keywords : upper Magdalenian, mobile art, stratigraphic context, bone industry, bone technology, Pyrénées occidentales 


\section{AUTEURS}

\section{LISE AURIÈRE}

Université Toulouse-II-le Mirail,UMR 5608 - TRACES - Maison de la recherche, 5 allée Antonio Machado, FR-31058 TOULOUSE cedex - lise.auriere@gmail.com

\section{FRANÇOIS-XAVIER CHAUVIÈRE}

Office du Patrimoine et de l'Archéologie de Neuchâtel, section archéologie, Latenium, CH-2068 Hauterive - francois-xavier.chauviere@ne.ch

\section{FRÉDÉRIC PLASSARD}

Université Bordeaux 1, UMR 5199 - PACEA - avenue des Facultés, FR-33400 Talence -

frederic.plassard@wanadoo.fr

\section{CAROLE FRITZ}

Université Toulouse-II-le Mirail,UMR 5608 - TRACES - Maison de la recherche, 5 allée Antonio Machado, FR-31058 TOULOUSE cedex - carole.fritz@univ-tlse2.fr,

\section{MORGANE DACHARY}

Université Toulouse-II-le Mirail,UMR 5608 - TRACES - Maison de la recherche, 5 allée Antonio Machado, FR-31058 TOULOUSE cedex - morgane-dachary@orange.fr 